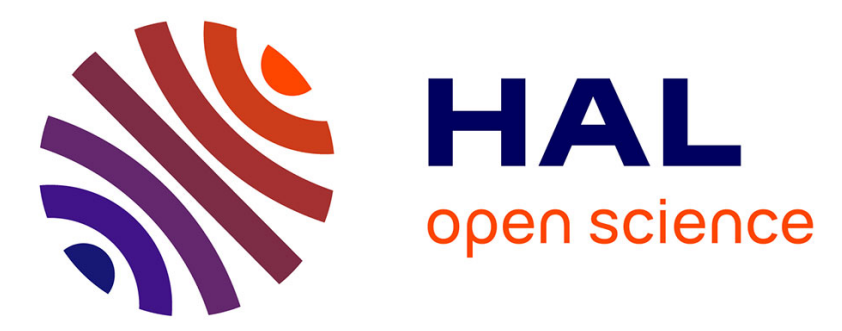

\title{
Numerical scheme for a water flow-driven forward stratigraphic model
}

Clément Cancès, Didier Granjeon, Quang-Huy Tran, Sylvie Wolf, Nicolas Peton

\section{- To cite this version:}

Clément Cancès, Didier Granjeon, Quang-Huy Tran, Sylvie Wolf, Nicolas Peton. Numerical scheme for a water flow-driven forward stratigraphic model. Computational Geosciences, 2020, 24, pp.37-60. 10.1007/s10596-019-09893-w . hal-01870347v2

\section{HAL Id: hal-01870347 \\ https://hal.science/hal-01870347v2}

Submitted on 22 Jun 2020

HAL is a multi-disciplinary open access archive for the deposit and dissemination of scientific research documents, whether they are published or not. The documents may come from teaching and research institutions in France or abroad, or from public or private research centers.
L'archive ouverte pluridisciplinaire HAL, est destinée au dépôt et à la diffusion de documents scientifiques de niveau recherche, publiés ou non, émanant des établissements d'enseignement et de recherche français ou étrangers, des laboratoires publics ou privés. 


\title{
Numerical scheme for a water flow-driven forward stratigraphic model
}

\author{
Nicolas Peton · Clément Cancès · Didier Granjeon · Quang-Huy Tran · Sylvie Wolf
}

Received: September 1, 2018 / Accepted: date

\begin{abstract}
This paper is concerned with extending the stratigraphic model previously introduced by Eymard et al. [Int. J. Numer. Methods Engrg. 60, 527-548 (2004)] and subsequently studied by Gervais and her coauthors for the simulation of large scale transport processes of sediments, subject to an erosion constraint. Two major novelties are considered: (i) the diffusion law relating the flux of sediments and the slope of the topography is now nonlinear and involves a $p$ Laplacian with $p>2$ in order for landscape evolutions to be more realistic; (ii) the sediment transport is now intertwined with the water flows due to lakes and rivers via a direct coupling at the continuous PDE level, which avoids empirical algorithms at the discrete level such as MFD (Multiple Flow Directions) at the price of additional $p$-Laplacians. Aimed at enriching the capabilities of IFPEN's simulator, these sophistications entail the construction of a new finite volume scheme, the details of which are supplied. The physical model is validated through several test cases. Finally, a further extension of the model to the case of multiple lithologies is presented, along with numerical results.
\end{abstract}

Keywords stratigraphic forward modeling - weatherlimited erosion $\cdot p$-Laplacian $\cdot$ complementarity problem

\section{Introduction}

The knowledge of subsoil structure lies at the heart of various fields, such as $\mathrm{CO}_{2}$ sequestration, management of water resources, exploitation of geothermal energy, where a

Clément Cancès

Inria, Univ. Lille, CNRS, UMR 8524 - Laboratoire Paul Painlevé, F59000. E-mail: clement.cances@inria.fr

Nicolas Peton · Didier Granjeon · Quang-Huy Tran · Sylvie Wolf IFP Energies nouvelles. 1 \& 4 avenue de Bois Préau, 92852 RueilMalmaison Cedex, France. E-mail: firstname.name@ifpen.fr detailed description of sediment layers geometry and their composition (sands, shales...) is often required. The ability to trace back the geological history of sedimentary basins is thus a key asset for the study of energetic georesources.

Sedimentary basins can be seen as large areas (hundreds of squared kilometers) where sediments due to erosion or other sources keep accumulating. Generally located in marine domains, some may also be found on the continent. Over large periods of time (millions of years), this leads to the formation of sediment layers, whose composition may vary according to environmental conditions.

The physical processes that account for the evolution of sedimentary basins are numerous and complex, but mainly depend on three parameters. The first one is the accommodation, that is, the available space for sedimentation. It is a direct consequence of tectonic processes, subsidence (downward motion of the earth's surface) and eustatism (sea level variations). The second one is the sediment supplies. These originate from erosion of neighbouring mountains, rivers bed load, or may be produced in situ (carbonates, corals). The third one is the sediment transport. Here, gravity plays an essential role, but rivers and submarine currents are also crucially involved.

The study the above processes over large time scales is the goal of stratigraphic modeling. For this purpose, geologists have two kinds of field data at their disposal. On one side, we have seismic data obtained by recording the reflection of waves emitted at the earth's surface. These enable us to estimate large areas but may not be accurate enough. On the other side, we have well-log data associated with a few given wells. These allow us to identify the type of sediments, along with their physical properties. Using these field data, a geological model can be designed in order to rebuild the infill scenario of the basin.

However, in complex or poorly known configurations (either by lack of data or low resolution), classical interpo- 
lation techniques reach their limit and it becomes necessary to resort to other approaches. Numerical simulation then appears as a suitable tool to test different infill scenarios of sedimentary basins and to find the best match with field data. As many hypothetical configurations have to be tried, it is vital that the stratigraphic model selected for simulation be efficient, both in terms of realism and computational time.

The stratigraphic model implemented in IFPEN's software DionisosFlow dates back to the nineties with the founding principles set up by Granjeon [24], notably regarding the maximal erosion rate. Granjeon's discrete algorithm for the erosion constraint was later reformulated by Eymard et al. [16] as the explicit discretization of a continuous PDE system in which the erosion constraint takes the form of a complementarity equation. This interpretation paved the way for an implicit discretization allowing for much larger time-steps, undertaken afterwards by Gervais [20] (see also $[13,14,21,22]$ for related mathematical aspects). Thanks to this achievement DionisosFlow remained competitive for about a decade, until it was decided to endow the code with two advanced physical phenomena.

The first novelty of interest is the tracking of knickpoints, which are commonly observed in natural landscape evolutions. This is only possible if the underlying diffusion process occurs at finite speed. Propagation at finite speed can in turn be guaranteed by some kind of nonlinear dependence of the sediment flux with respect to the gradient of the topography. By "some kind of nonlinear dependence," we mean that the sediment flux is proportional to $|\nabla h|^{p-2} \nabla h$ - where $h$ denotes the height of sediments and $p>2$ - instead of $\nabla h$ as is the case in [20]. The appearance of the $p$-Laplacian makes the Two-Point Flux Approximation of [20] obsolete. To correctly approximate $|\nabla h|^{p-2}$, Cancès et al. [7] (see also Peton [28]) sketched out a solution inspired from the work of Andreianov et al. [1]. We take the opportunity of this paper to provide full details of the new scheme.

The second novelty of interest is the water effects associated with the impact of rivers and lakes. In this respect several discrete algorithms, known as Multiple Flow Directions (MFD), are available (see Freeman [19], Holmgren [25], Quinn et al. [29] or Tarboton [32]). However, they suffer from many drawbacks: apart from being non-parallelizable and highly sensitive to the grid orientation effect, none of them has a clear limit as the mesh size goes to zero. Put another way, none of them can be understood as a discretization of some continuous PDE system. Here, the situation is worse than what happened with the discrete algorithm for the erosion constraint, where such an interpretation was possible. For all these reasons, we advocate to give up the MFD algorithms and to look for a direct coupling between sediment transport and water effects at the continuous level, based on fluid mechanics and empirical laws from sedimentology. The model we propose involves two additional $p$ -
Laplacians, one with $p=3 / 2$ and the other with $p=5 / 2$. It bears strong analogies with but remains differ from that of Birnir et al. [6]. Let us also mention a few remote works that rest upon a similar coupling philosophy but the scales of which are much smaller than ours: Delestre et al. [9] on rainwater overland-flows, Cordier et al. [8] on bedload transport, and Audusse et al. [2] on sedimentary river beds.

This paper is outlined as follows. In $\S 2$, we derive the stratigraphic model in the case of one lithology. This model takes into account the nonlinear sediment diffusion and the water flows, in addition to the preexisting erosion constraint. Some properties of the model are enumerated in $\S 3$. The construction of an appropriate Finite Volume scheme is addressed in $\S 4$, where as a preliminary step we focus on the unsteady $p$-Laplacian equation in order to highlight the intrinsic difficulties of the problem. Unlike Gervais [20], we recommend the Newton-min method to solve the overall algebraic system arising at each time-step, in place of the $v a$ riable switching procedure. Some properties of the scheme are elaborated on in $\S 5$, among which the existence of a discrete solution. In $\S 6$, numerical results corresponding to two test cases are analyzed and commented on. Finally, in $\S 7$, we extend the model to the multi-lithology case, where most of the methods developed for the single-lithology case are easily carried over. Notwithstanding, specific features need to be handled, such as the sediment columns and their discretization.

\section{Derivation of the stratigraphic model}

We start by revisiting some fundamentals of fluid mechanics in $\$ 2.1$, in order to bring out reliable approximations for our problem. Since the emphasis is laid mostly on the coupling of sediment transport with water effects, the treatment of the erosion rate is postponed to $\S 2.2$. The reader uninterested in the derivation is referred to $\S 2.3$, where the full model is stated.

\subsection{Approximate balance laws}

In a first stage, we put aside gravity diffusion and look for two conservation laws: one for the water height (denoted by $\widetilde{h}$ ), and the other for the sediment height (denoted by $h$ ).

Let us consider a water flow in a channel. Based on the approach of Birnir et al. [6] for modeling long time approximations of water flows, our analysis takes the shallow water equations with friction

$$
\begin{array}{ll}
\partial_{t} \widetilde{h}+\operatorname{div}(\widetilde{h} \widetilde{\mathbf{u}}) & =0, \\
\partial_{t}(\widetilde{h} \widetilde{\mathbf{u}})+\operatorname{div}(\widetilde{h} \widetilde{\mathbf{u}} \otimes \widetilde{\mathbf{u}})+g \widetilde{h} \nabla(\widetilde{h}+h) & =-C|\widetilde{\mathbf{u}}| \widetilde{\mathbf{u}},
\end{array}
$$




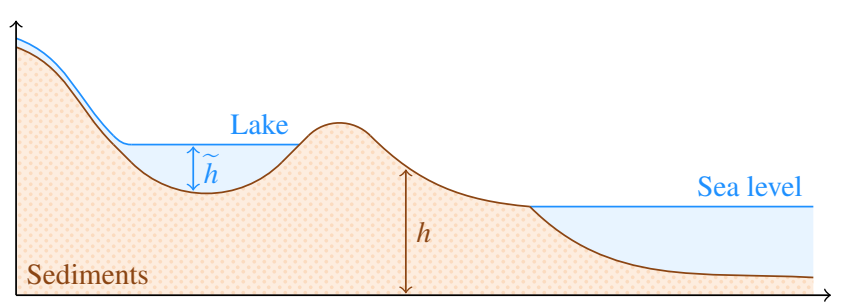

Fig. 1: Notations for the stratigraphic model: $\widetilde{h}$ denotes the water height and $h$ the sediment height.

as a starting point, where $\widetilde{\mathbf{u}}$ represents the water speed, $g$ denotes the acceleration due to gravity, and $C$ is the friction coefficient. The latter is assumed to be a constant. Introducing two characteristic heights $\widetilde{H}, H$ (hundreds of meters), a characteristic length $L$ (hundreds of kilometers), a characteristic time $T$ (millions of years), we proceed to nondimensionalize (1) by defining the nondimensional variables

$\check{\widetilde{h}}=\frac{\widetilde{h}}{\widetilde{H}}, \quad \check{h}=\frac{h}{H}, \quad \check{\widetilde{\mathbf{u}}}=\frac{\widetilde{\mathbf{u}}}{\widetilde{U}}, \quad \check{x}=\frac{x}{L}, \quad \check{y}=\frac{y}{L}, \quad \check{t}=\frac{t}{T}$,

where $\widetilde{U}=L / T$ is the characteristic speed. It is readily checked that system (1) is equivalent to

$\partial_{\tilde{t}} \check{\widetilde{h}}+\operatorname{div}(\check{\widetilde{h}} \check{\widetilde{\mathbf{u}}})$ $=0$,

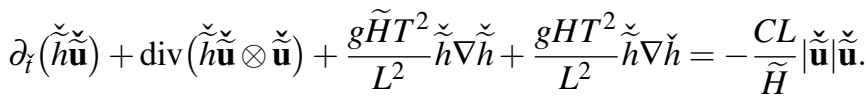

For sedimentary basins, the water and sediment heights are usually small compared to the domain size. More precisely, the flow regime is such that we have

$\frac{g \widetilde{H} T^{2}}{L^{2}} \gg 1, \quad \frac{g H T^{2}}{L^{2}} \gg 1, \quad \frac{C L}{\widetilde{H}} \gg 1$.

This suggests to neglect the inertia terms in the nondimensionalized momentum balance, which is commonly known as hydrostatic approximation. Returning to its dimensionalized counterpart (1b), the equilibrium of the driving forces reads

$|\widetilde{\mathbf{u}}| \widetilde{\mathbf{u}}=-\widetilde{K}^{2} \widetilde{h} \nabla(\widetilde{h}+h)$.

Here $\widetilde{K}^{2}=g / C$ can be seen as the Chézy coefficient [23]. By inverting formula (2), we can express the water speed as

$\widetilde{\mathbf{u}}=-\widetilde{K} \boldsymbol{h}^{1 / 2}|\nabla(\widetilde{h}+h)|^{-1 / 2} \nabla(\widetilde{h}+h)$.

Then, combining equations (1a) and (3), we obtain

$\partial_{t} \widetilde{h}+\operatorname{div}\left(-\widetilde{K} \tilde{h}^{3 / 2}|\nabla(\widetilde{h}+h)|^{-1 / 2} \nabla(\widetilde{h}+h)\right)=0$.

which is the desired conservation law for the water height.

Next, we switch to sediments. Contrary to the case of water, we will use empirical laws. These are described in various textbooks (see, e.g., Granjeon [24]) and stipulate that the sediment flux $\mathbf{F}$ is proportional to the shear stress due to water (denoted by $\tau$ ) to the power $3 / 2$, i.e.,

$\mathbf{F}=k|\boldsymbol{\tau}|^{1 / 2} \boldsymbol{\tau}$,

where $k$ is a constant. Here we suppose that the water flow is strong enough to induce a constant sediment flow, hence we do not consider critical shear stress in equation (5). Since we are working over large time and space scales, the stress $\tau$ takes the form

$\tau=-\rho g \tilde{h} \nabla(\widetilde{h}+h)$

where $\rho$ represents the water density and $g$ is the acceleration due to gravity. By plugging (6) into (5), we end up with the sediment flux

$$
\mathbf{F}=-k(\rho g)^{3 / 2} \widetilde{h}^{3 / 2}|\nabla(\widetilde{h}+h)|^{1 / 2} \nabla(\widetilde{h}+h) .
$$

For practical reasons, we choose to use a further approximation. Once again, as we are working over large time scales, water flows along slopes with only thin streams. In other words, the gradient of the total height $\nabla(\widetilde{h}+h)$ is almost similar to $\nabla h$, except for regions containing lakes. However, in that specific case, we suppose for the sake of simplicity that the sediment flow follows the topography slope, namely, $\nabla h$. This approximation yields

$\mathbf{F}=-K \widetilde{h}^{3 / 2}|\nabla(\widetilde{h}+h)|^{1 / 2} \nabla h$

as the new definition of the sediment flux, with $K=k(\rho g)^{\frac{3}{2}}$.

In a second stage, we recover gravity diffusion by adding an extra term to definition (7) of the sediment flux. The new sediment flux reads

$\mathbf{F}=-K(h) \widetilde{h}^{3 / 2}|\nabla(\widetilde{h}+h)|^{1 / 2} \nabla h-K_{g}(h)|\nabla h|^{p-2} \nabla h$,

and the desired conservation law for the sediment height is

$\partial_{t} h+\operatorname{div} \mathbf{F}=0$

The exponent $p$ in (8) must be greater than 2 to ensure finite propagation speed $[4,26]$. As functions of the sediment height $h$, the diffusion coefficients $K$ and $K_{g}$ often take two values so as to mark the difference between continental and marine domains, that is,

$$
\begin{gathered}
K(h)= \begin{cases}K_{m}, & \text { if } h<H_{m}, \\
K_{c}, & \text { if } h \geqslant H_{m},\end{cases} \\
K_{g}(h)= \begin{cases}K_{g, m}, & \text { if } h<H_{m}, \\
K_{g, c}, & \text { if } h \geqslant H_{m},\end{cases}
\end{gathered}
$$

where $H_{m}$ represents the (known) sea level. 


\subsection{Maximal erosion rate}

The balance laws (4) and (9) are not sufficient to faithfully reflect the evolution of sedimentary basins. Indeed, according to geomorphologists, sedimentation $\left(\partial_{t} h>0\right)$ and erosion $\left(\partial_{t} h<0\right)$ are non-symmetric processes: soil material must first be produced in situ by weathering process prior to being transported by diffusion. Granjeon [24] postulated that the erosion is limited from below by $-E$, where $E>0$ is a known maximal erosion rate depending on the climate, the type of sediments, and the bathymetry, so that

$\partial_{t} h+E \geqslant 0$.

To impose this constraint while preserving conservativity of the sediment height, Eymard et al. [16] introduced a new variable $\lambda \in(0,1]$ called flux limiter and modified (9) as

$\partial_{t} h+\operatorname{div}(\lambda \mathbf{F})=0$,

Locally, either the erosion constraint is saturated $\left(\partial_{t} h+E=\right.$ $0)$ or the flux is unlimited $(\lambda=1)$. This is best formulated by means of the complementarity condition

$1-\lambda \geqslant 0, \quad \partial_{t} h+E \geqslant 0, \quad(1-\lambda)\left(\partial_{t} h+E\right)=0$,

which can be further condensed into the more convenient equation

$\min \left(1-\lambda, \partial_{t} h+E\right)=0$.

Intuitively, this constraint can be viewed as a way to introduce some a priori information on the availability of the sediment. As illustrated in the two lower panels of Figure 2, as soon as the maximal amount of sediment $E \Delta t$ available for the time-step has been "consumed" somewhere, the diffusion process abruptly stops at this place.

\subsection{Full model}

The stratigraphic model we will study is made up of the two previous conservation laws and the constraint on the erosion rate. In equation (13), the second argument of the min function is replaced by $E-\operatorname{div}(\lambda \mathbf{F})$ to eliminate the time derivative. Let $\Omega \subset \mathbb{R}^{2}$ be an open connected bounded domain representing the basin. With a slight abuse of notation, the symbol $T$ now stands for the simulation time. The unknowns $\widetilde{h}, h$ and $\lambda$ are functions of $(\mathbf{x}, t) \in \Omega \times[0, T]$ and solve

$$
\begin{array}{lll}
\partial_{t} \widetilde{h}+\operatorname{div}(\widetilde{\mathbf{F}}) & =0, & \text { on } \Omega \times[0, T], \\
\partial_{t} h+\operatorname{div}(\lambda \mathbf{F}) & =0, & \text { on } \Omega \times[0, T], \\
\min (1-\lambda, E-\operatorname{div}(\lambda \mathbf{F}))=0, & \text { on } \Omega \times[0, T],
\end{array}
$$

where the fluxes are written under the form

$$
\begin{aligned}
& \widetilde{\mathbf{F}}=-\widetilde{K}\left(\widetilde{h}^{+}\right)^{3 / 2}|\nabla(\widetilde{h}+h)|^{-1 / 2} \nabla(\widetilde{h}+h), \\
& \mathbf{F}=-\left(\widetilde{h}^{+}\right)^{3 / 2}|\nabla(\widetilde{h}+h)|^{1 / 2} \nabla \psi(h)-|\nabla h|^{p-2} \nabla \psi_{g}(h),
\end{aligned}
$$

with the functions

$$
\begin{aligned}
\psi(h) & =\int_{H_{m}}^{h} K(a) \mathrm{d} a, \\
\psi_{g}(h) & =\int_{H_{m}}^{h} K_{g}(a) \mathrm{d} a .
\end{aligned}
$$

In the definition of the fluxes (15), positive parts have been introduced in order to raise $\widetilde{h}$ to the power $3 / 2$. This is necessary, even though we expect this quantity to remain nonnegative. Furthermore, this alteration will be useful at the discrete level to avoid singularities (e.g., while using Newton's method). To close the physical model, we prescribe Neumann-type boundary conditions corresponding to water and sediment inflows as

$\widetilde{\mathbf{F}} \cdot \mathbf{n}=\widetilde{\phi} \leqslant 0, \quad$ on $\partial \Omega \times[0, T]$,

$\mathbf{F} \cdot \mathbf{n}=\phi \leqslant 0, \quad$ on $\partial \Omega \times[0, T]$.

Finally, initial states are given by

$\left.\widetilde{h}\right|_{t=0}=\widetilde{h}^{0}, \quad$ on $\Omega$,

$\left.h\right|_{t=0}=h^{0}$, on $\Omega$,

with $0 \leqslant \widetilde{h}^{0} \leqslant \widetilde{h}^{*}$ and $h_{*} \leqslant h^{0} \leqslant h^{*}$ for some $h_{*}, h^{*}, \widetilde{h}^{*} \in \mathbb{R}$.

\section{Basic properties of the model}

Before thinking about a numerical scheme for the model (14)-(18), it is helpful to enumerate some of its properties at the continuous level. Let us assume that $\tilde{h}, h$ and $\lambda$ are smooth solutions in order to justify the calculations below. We also admit that the flux limiter $\lambda$ is positive, which will be proven at the discrete level in Lemma 4.

\section{Lemma 1 (Nonnegativity of the water height) The water} height is nonnegative, i.e.,

$\widetilde{h} \geqslant 0 \quad$ in $\Omega \times \mathbb{R}_{+}$.

Proof Let $\mathrm{T} \in[0, T]$. Multiplying the conservation law (14a) by the negative part $\widetilde{h}^{-}$and integrating over $\Omega \times[0, \mathrm{~T}]$ yields

$$
\begin{aligned}
\int_{0}^{\mathrm{T}} \int_{\Omega} \widetilde{h}^{-} \partial_{t} \widetilde{h} \mathrm{~d} x \mathrm{~d} y \mathrm{~d} t+\int_{0}^{\mathrm{T}} \int_{\Omega} \widetilde{h}^{-} \operatorname{div}\left(-\widetilde{K}\left(\widetilde{h}^{+}\right)^{3 / 2}\right. \\
\left.\times|\nabla(\widetilde{h}+h)|^{-1 / 2} \nabla(\widetilde{h}+h)\right) \mathrm{d} x \mathrm{~d} y \mathrm{~d} t=0 .
\end{aligned}
$$

In the first integral, we notice that $\widetilde{h}^{-} \partial_{t} \widetilde{h}=\partial_{t}\left[\frac{1}{2}\left(\widetilde{h}^{-}\right)^{2}\right]$. In the second integral, we apply the Green theorem and invoke 

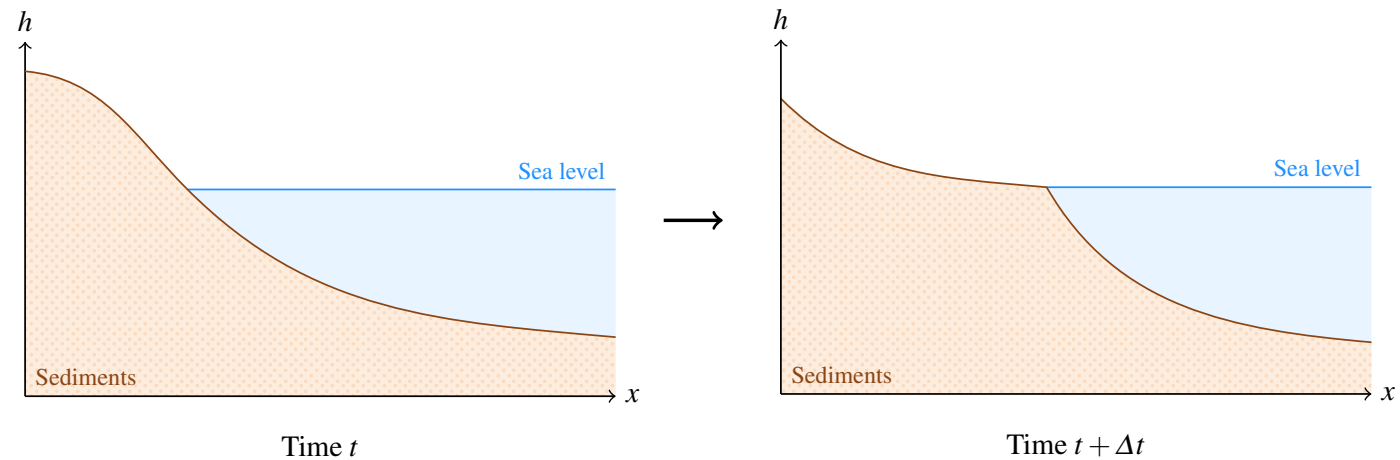

(a) Evolution of the system without constraint.
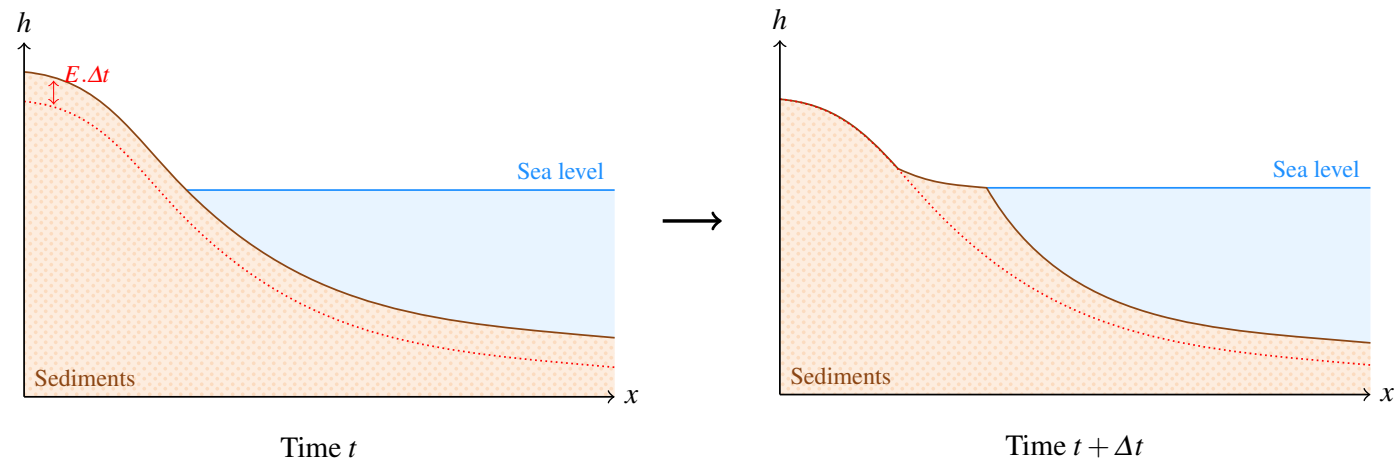

(b) Evolution of the system with constraint.

Fig. 2: Comparison of the system evolution with and without constraint on the erosion process.

the fact $\widetilde{h}^{-}\left(\widetilde{h}^{+}\right)^{3 / 2}$ and $\nabla \widetilde{h}^{-}\left(\widetilde{h}^{+}\right)^{3 / 2}$ vanish identically. As a result, equation (20) boils down to

$\int_{0}^{\mathrm{T}} \frac{\mathrm{d}}{\mathrm{d} t} \int_{\Omega} \frac{1}{2}\left(\widetilde{h}^{-}\right)^{2} \mathrm{~d} x \mathrm{~d} y=\left.\int_{\Omega} \frac{1}{2}\left(\widetilde{h}^{-}\right)^{2}\right|_{t=\mathrm{T}} \mathrm{d} x \mathrm{~d} y=0$,

since the initial water height is nonnegative. From this formula, we infer that $\widetilde{h}^{-}(\cdot, \mathrm{T})=0$ on $\Omega$.

Lemma 2 (Estimate on the sediment height) The sediment height is uniformly bounded from below, i.e.,

$h \geqslant h_{*} \quad$ in $\Omega \times \mathbb{R}_{+}$.

Proof Let $\mathrm{T} \in[0, T]$. Multiplying the conservation law (14b) by the negative part $\left(h-h_{*}\right)^{-}$and integrating over $\Omega \times[0, \mathrm{~T}]$ yields

$\int_{0}^{\mathrm{T}} \int_{\Omega}\left(h-h_{*}\right)^{-} \partial_{t} h \mathrm{~d} x \mathrm{~d} y \mathrm{~d} t$

$$
+\int_{0}^{\mathrm{T}} \int_{\Omega}\left(h-h_{*}\right)^{-} \operatorname{div}(\lambda \mathbf{F}) \mathrm{d} x \mathrm{~d} y \mathrm{~d} t=0 .
$$

In the first integral, we notice that $\left(h-h_{*}\right)^{-} \partial_{t} h=\partial_{t}\left[\frac{1}{2}(h-\right.$ $\left.\left.h_{*}\right)^{-}\right]^{2}$. In the second second, we apply the Green theorem. Expanding the formula of the sediment flux and arguing as in Lemma 1, we end up with

$\left.\int_{\Omega} \frac{1}{2}\left[\left(h-h_{*}\right)^{-}\right]^{2}\right|_{t=\mathrm{T}} \mathrm{d} x \mathrm{~d} y \leqslant 0$.
This implies $h(\cdot, \mathrm{T}) \geqslant h_{*}$ over $\Omega$.

Lemma 3 (Mass conservation of water and sediments) At any time $\mathrm{T} \in[0, T]$, the water and sediment heights satisfy

$\left.\int_{\Omega} \widetilde{h}\right|_{t=\mathrm{T}} \mathrm{d} x \mathrm{~d} y=\int_{\Omega} \widetilde{h}_{0} \mathrm{~d} x \mathrm{~d} y-\int_{0}^{\mathrm{T}} \int_{\partial \Omega} \widetilde{\phi} \mathrm{d} \sigma \mathrm{d} t$,

$\left.\int_{\Omega} h\right|_{t=\mathrm{T}} \mathrm{d} x \mathrm{~d} y=\int_{\Omega} h_{0} \mathrm{~d} x \mathrm{~d} y-\int_{0}^{\mathrm{T}} \int_{\partial \Omega} \phi \mathrm{d} \sigma \mathrm{d} t$.

Proof Straightforward by integrating (14a)-(14b) over $\Omega \times$ $[0, \mathrm{~T}]$ and by using the Stokes formula.

\section{Finite Volume discretization}

To compute an approximate solution of (14)-(18), our task to devise a Finite Volume scheme that correctly takes into account various $p$-Laplacians and that coincides as much as possible with the method of $[13,14,21,22]$ for $p=2$ and without any water. To clearly perceive the discretization of a $p$-Laplacian, we first focus on a simpler configuration, namely, the unsteady $p$-Laplacian equation.

\subsection{Notations}

The rectangular domain $\Omega=\left[0, L_{x}\right] \times\left[0, L_{y}\right]$ is discretized into $N_{x} \times N_{y}$ uniform cells of size $\Delta x \times \Delta y=L_{x} / N_{x} \times L_{y} / N_{y}$, 
where $N_{x}, N_{y}$ are two positive integers. The cell $(i, j)$, denoted by $\mathscr{C}_{i, j}$, is centered at $\mathbf{x}_{i j}=((i-1 / 2) \Delta x,(j-1 / 2) \Delta y)$. The boundary $\partial \Omega$ of the domain is divided into four parts $\Gamma_{b}, \Gamma_{t}, \Gamma_{l}$ and $\Gamma_{r}$, as depicted in Figure 3 . Time is discretized by $0=t^{0}<t^{1}<\cdots<t^{n}<\ldots$, using the time-steps $\Delta t^{n}=$ $t^{n+1}-t^{n}$.

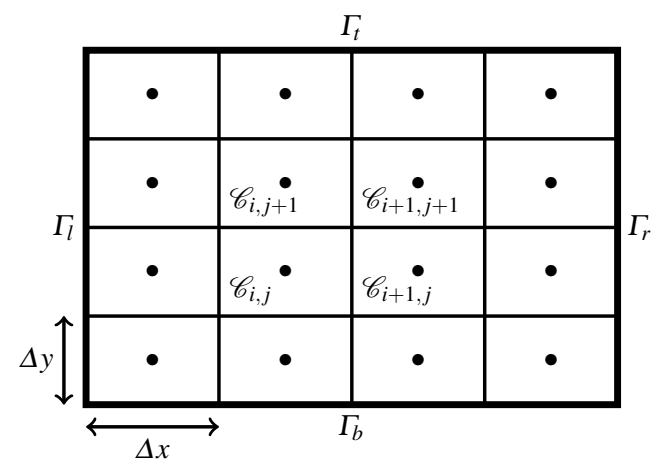

Fig. 3: Finite Volume notations.

\subsection{A simpler case: the unsteady $p$-Laplacian equation}

The unsteady $p$-Laplacian equation contains a core difficulty of system (14)-(18). The unknown $h: \Omega \times \mathbb{R}_{+} \rightarrow \mathbb{R}$ is momentarily assumed to solve the PDE

$\partial_{t} h+\operatorname{div} \check{\mathbf{F}}=0$,

with the flux

$\check{\mathbf{F}}=-|\nabla h|^{p-2} \nabla h, \quad p>1$.

It is known that the elementary solution, called Barenblatt [4], behaves differently $[10,26]$ according to whether the exponent $p$ is greater or less than 2, as illustrated in Figure 4. If $p>2$ the solution is compactly supported and the diffusion speed is finite. If $p<2$ the solution does not have a compact support at each time $t \geqslant 0$ and the diffusion speed is infinite. The linear case $p=2$ is the classical heat equation.

In the Finite Volume context, the principal difficulty lies in approximating the term $|\nabla h|^{p-2}$ at each interface between the cells. Over rectangular meshes, Andreianov et al. [1] has developped a method for the stationary $p$-Laplacian equation. Here we adapt their method to the unsteady version by first computing an approximation of $\left|\nabla h^{n}\right|^{2}$ on each dual cell (cf. Figure 5) using the formula

$$
\begin{aligned}
B_{i+1 / 2, j+1 / 2}^{n}= & \frac{1}{2}\left(\frac{h_{i+1, j}^{n}-h_{i, j}^{n}}{\Delta x}\right)^{2}+\frac{1}{2}\left(\frac{h_{i+1, j+1}^{n}-h_{i, j+1}^{n}}{\Delta x}\right)^{2} \\
& +\frac{1}{2}\left(\frac{h_{i, j+1}^{n}-h_{i, j}^{n}}{\Delta y}\right)^{2}+\frac{1}{2}\left(\frac{h_{i+1, j+1}^{n}-h_{i+1, j}^{n}}{\Delta y}\right)^{2},
\end{aligned}
$$

then by raising the result to the power $(p-2) / 2$ to get an approximation of $\left|\nabla h^{n}\right|^{p-2}$. It is worth noticing that the approximation (25) is coercive, i.e., it cannot vanish unless the four values on the dual cell are identical. Coercivity holds naturally without any stabilization, as is customarily required in other Finite Volume methods for nonlinear elliptic problems, such as those proposed by Droniou [12] and Eymard et al. [18] over general meshes. This is a significant advantage of Andreianov et al.'s scheme.

The discrete flux at each inner edge of the domain is given, in the case $p>2$, by the semi-implicit formulae

$$
\begin{aligned}
\check{F}_{i+1 / 2, j}^{n+1}=-\frac{1}{2}\left[\left(B_{i+1 / 2, j-1 / 2}^{n}\right)^{p / 2-1}\right. & \left.+\left(B_{i+1 / 2, j+1 / 2}^{n}\right)^{p / 2-1}\right] \\
& \times \frac{h_{i+1, j}^{n+1}-h_{i, j}^{n+1}}{\Delta x}, \\
\check{F}_{i, j+1 / 2}^{n+1}=-\frac{1}{2}\left[\left(B_{i-1 / 2, j+1 / 2}^{n}\right)^{p / 2-1}\right. & \left.+\left(B_{i+1 / 2, j+1 / 2}^{n}\right)^{p / 2-1}\right] \\
& \times \frac{h_{i, j+1}^{n+1}-h_{i, j}^{n+1}}{\Delta y} .
\end{aligned}
$$

The choice of keeping the approximation of $|\nabla h|^{p-2}$ explicit is motivated by saving computational cost. Numerical experiments testify to the fact that the semi-implicit scheme compares well with the full implicit one. If $p<2$, a regularization is needed to avoid singularities. In that case, $B_{i+1 / 2, j+1 / 2}^{n}$ is replaced by $\max \left(B_{i+1 / 2, j+1 / 2}^{n}, \varepsilon\right)$, with $\varepsilon>0$ small. For all $p>1$, the Finite Volume scheme reads

$\frac{h_{i, j}^{n+1}-h_{i, j}^{n}}{\Delta t}+\frac{\check{F}_{i+1 / 2, j}^{n+1}-\check{F}_{i-1 / 2, j}^{n+1}}{\Delta x}+\frac{\check{F}_{i, j+1 / 2}^{n+1}-\check{F}_{i, j-1 / 2}^{n+1}}{\Delta y}=0$

for $1 \leqslant i \leqslant N_{x}$ and $1 \leqslant j \leqslant N_{y}$.

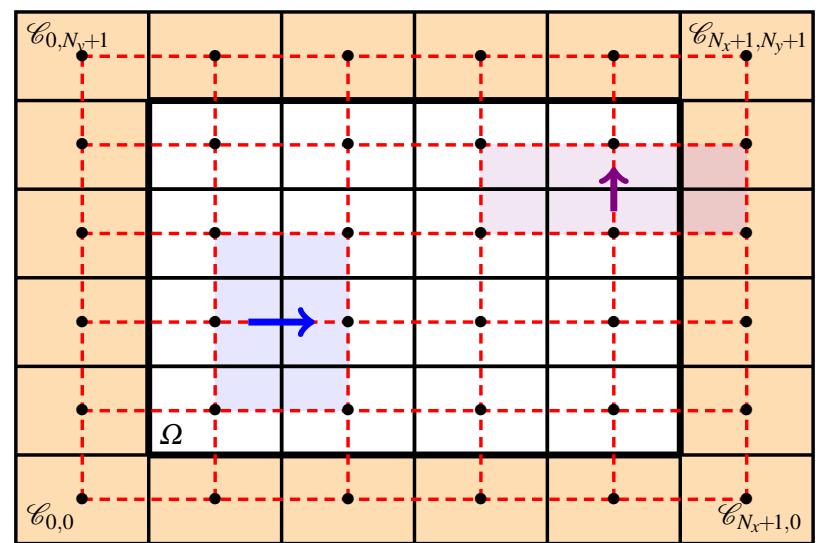

Fig. 5: Mesh illustration: initial domain $\Omega$ (in white), ghost cells (in orange) and dual mesh (in red). 


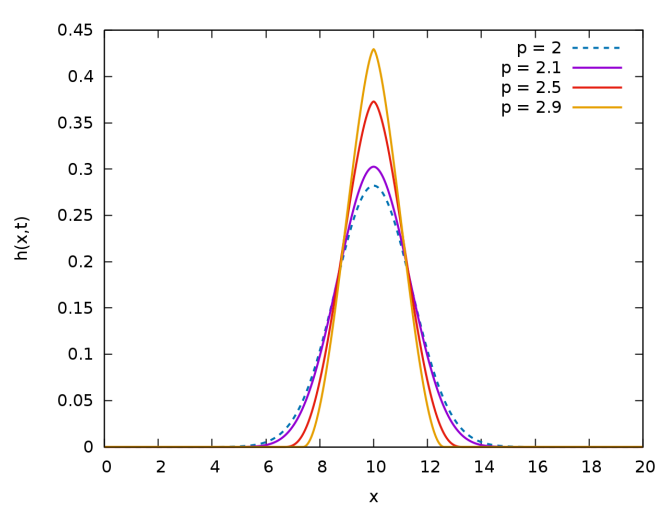

(a) Slow diffusion case $(p>2)$.

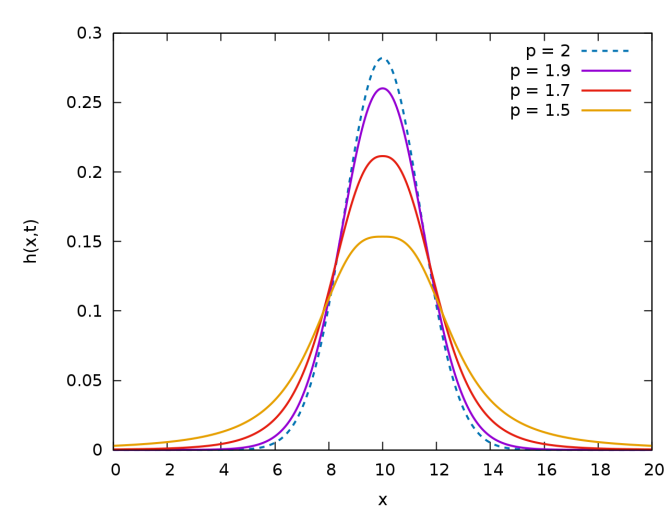

(b) Fast diffusion case $(p<2)$.

Fig. 4: Elementary solution in 1D at time $t=1$.

Since normal fluxes are prescribed on the boundary, it seems at first sight that the scheme (27) is well defined everywhere and there is no need to define the $B^{n}$ 's at the boundary. Nevertheless, this impression is dispelled upon close inspection of the fluxes tangential to the boundary. Let us take a look at the Figure 5. The approximation of the blue flux, associated to an inner edge, can be directly computed using (26). On the contrary, for the purple one which is tangential to the domain, we observe that data outside of $\Omega$ are needed to perform its approximation.

To remedy this issue, we consider a layer of ghost cells around the initial domain, and associate a new unknown $h$ to each of these cells. To have a well-posed system we then need to add as much equations as ghost cells. The idea is to link the value of the new unknowns with the fluxes prescribed at the boundary. For $1 \leqslant i \leqslant N_{x}, 1 \leqslant j \leqslant N_{y}$, the equations take the form

$$
\begin{aligned}
& \Gamma_{b}: \check{F}_{i, 1 / 2}^{n+1}+\frac{1}{\Delta x \Delta t} \int_{t^{n}}^{t^{n+1}} \int_{(i-1) \Delta x}^{i \Delta x} \phi(x, 0, t) \mathrm{d} x \mathrm{~d} t=0, \\
& \Gamma_{l}: \check{F}_{1 / 2, j}^{n+1}+\frac{1}{\Delta y \Delta t} \int_{t^{n}}^{t^{n+1}} \int_{(j-1) \Delta y}^{j \Delta y} \phi(0, y, t) \mathrm{d} y \mathrm{~d} t=0, \\
& \Gamma_{t}: \check{F}_{i, N_{y}+1 / 2}^{n+1}-\frac{1}{\Delta x \Delta t} \int_{t^{n}}^{t^{n+1}} \int_{(i-1) \Delta x}^{i \Delta x} \phi\left(x, L_{y}, t\right) \mathrm{d} x \mathrm{~d} t=0, \\
& \Gamma_{r}: \check{F}_{N_{x}+1 / 2, j}^{n+1}-\frac{1}{\Delta y \Delta t} \int_{t^{n}}^{t^{n+1}} \int_{(j-1) \Delta y}^{j \Delta y} \phi\left(L_{x}, y, t\right) \mathrm{d} y \mathrm{~d} t=0 .
\end{aligned}
$$

There remain four ghost cells at the corners $i \in\left\{0, N_{x}+1\right\}$, $j \in\left\{0, N_{y}+1\right\}$, to be taken care of. For simplicity, we choose to use an extrapolation of the neighbouring values, that is,

$$
\begin{array}{llll}
\mathscr{C}_{0,0}: & h_{0,0}^{n+1}+h_{1,1}^{n+1}-h_{1,0}^{n+1}-h_{0,1}^{n+1}=0, \\
\mathscr{C}_{N_{x}+1,0}: & h_{N_{x}+1,0}^{n+1}+h_{N_{x}, 1}^{n+1}-h_{N_{x}+1,1}^{n+1}-h_{N_{x}, 0}^{n+1}=0, \\
\mathscr{C}_{0, N_{y}+1}: & h_{0, N_{y}+1}^{n+1}+h_{1, N_{y}}^{n+1}-h_{1, N_{y}+1}^{n+1}-h_{0, N_{y}}^{n+1}=0, \\
\mathscr{C}_{N_{x}+1, N_{y}+1}: & h_{N_{x}+1, N_{y}+1}^{n+1}+h_{N_{x}, N_{y}}^{n+1}-h_{N_{x}, N_{y}+1}^{n+1}-h_{N_{x}+1, N_{y}}^{n+1}=0 .
\end{array}
$$

4.3 Numerical scheme for the stratigraphic model

We are now in a position to return to the stratigraphic model (14)-(18). Since we have $|\nabla h|$ and $|\nabla(\widetilde{h}+h)|$ in the model, we need not only a dual-cell approximation $B_{i+1 / 2, j+/ 12}^{n}$ for $\left|\nabla h^{n}\right|^{2}$ but also a dual-cell approximation $\bar{B}_{i+1 / 2, j+1 / 2}^{n}$ for $\left|\nabla\left(\widetilde{h}^{n}+h^{n}\right)\right|^{2}$. Following the spirit of (25), the latter approximation can be straightforwardly written as

$$
\begin{aligned}
\bar{B}_{i+1 / 2, j+1 / 2}^{n} & =\frac{1}{2}\left(\frac{\widetilde{h}_{i+1, j}^{n}+h_{i+1, j}^{n}-\widetilde{h}_{i, j}^{n}-h_{i, j}^{n}}{\Delta x}\right)^{2} \\
& +\frac{1}{2}\left(\frac{\widetilde{h}_{i+1, j+1}^{n}+h_{i+1, j+1}^{n}-\widetilde{h}_{i, j+1}^{n}-h_{i, j+1}^{n}}{\Delta x}\right)^{2} \\
& +\frac{1}{2}\left(\frac{\widetilde{h}_{i, j+1}^{n}+h_{i, j+1}^{n}-\widetilde{h}_{i, j}^{n}-h_{i, j}^{n}}{\Delta y}\right)^{2} \\
& +\frac{1}{2}\left(\frac{\widetilde{h}_{i+1, j+1}^{n}+h_{i+1, j+1}^{n}-\widetilde{h}_{i+1, j}^{n}-h_{i+1, j}^{n}}{\Delta y}\right)^{2} .
\end{aligned}
$$

\subsubsection{Conservation law for water}

To discretize the conservation law for the water height (14a), we first recast it in a slightly different way, as

$\partial_{t} \widetilde{h}+\operatorname{div}\left[\left(\widetilde{h}^{+}\right)^{3 / 2} \widetilde{\mathbf{G}}\right]=0$,

with

$\widetilde{\mathbf{G}}=-\widetilde{K}|\nabla(\widetilde{h}+h)|^{-1 / 2} \nabla(\widetilde{h}+h)$.

The idea behind (29) is to upwind the "transported" quantity $\left(\widetilde{h}^{+}\right)^{3 / 2}$ with respect to the sign of the normal "velocity" $\widetilde{G}=\widetilde{\mathbf{G}} \cdot \mathbf{n}$ at the edges. This amounts to defining the discrete 
water fluxes

$\widetilde{F}_{i+1 / 2, j}^{n+1}=\left[\left(\widetilde{h}_{i, j}^{n+1}\right)^{+}\right]^{3 / 2}\left(\widetilde{G}_{i+1 / 2, j}^{n+1}\right)^{+}+\left[\left(\widetilde{h}_{i+1, j}^{n+1}\right)^{+}\right]^{3 / 2}\left(\widetilde{G}_{i+1 / 2, j}^{n+1}\right)^{-}$ $\widetilde{F}_{i, j+1 / 2}^{n+1}=\left[\left(\widetilde{h}_{i, j}^{n+1}\right)^{+}\right]^{3 / 2}\left(\widetilde{G}_{i, j+1 / 2}^{n+1}\right)^{+}+\left[\left(\widetilde{h}_{i, j+1}^{n+1}\right)^{+}\right]^{3 / 2}\left(\widetilde{G}_{i, j+1 / 2}^{n+1}\right)^{-}$,

where $a^{+}=\max (a, 0)$ and $a^{-}=\min (a, 0)$. To define the $\widetilde{G}$ 's, we need an approximation of $|\nabla(\widetilde{h}+h)|^{-1 / 2}$. This can be achieved by raising formula (28) to the power $-1 / 4$. Because of the negative exponent, a regularization must be performed in order to avoid infinities in flat zones, which results in

$$
\begin{aligned}
& \widetilde{G}_{i+1 / 2, j}^{n+1}=-\widetilde{K}\left[\max \left(\bar{B}_{i+1 / 2, j-1 / 2}^{n}, \varepsilon\right)^{-1 / 4}\right. \\
& \left.+\max \left(\bar{B}_{i+1 / 2, j+1 / 2}^{n}, \varepsilon\right)^{-1 / 4}\right] \frac{\widetilde{h}_{i+1, j}^{n+1}+h_{i+1, j}^{n+1}-\widetilde{h}_{i, j}^{n+1}-h_{i, j}^{n+1}}{2 \Delta x}, \\
& \widetilde{G}_{i, j+1 / 2}^{n+1}=-\widetilde{K}\left[\max \left(\bar{B}_{i-1 / 2, j+1 / 2}^{n}, \varepsilon\right)^{-1 / 4}\right. \\
& \left.+\max \left(\bar{B}_{i+1 / 2, j+1 / 2}^{n}, \varepsilon\right)^{-1 / 4}\right] \frac{\widetilde{h}_{i, j+1}^{n+1}+h_{i, j+1}^{n+1}-\widetilde{h}_{i, j}^{n+1}-h_{i, j}^{n+1}}{\Delta y} .
\end{aligned}
$$

Finally, the interior scheme is given by

$$
\frac{\widetilde{h}_{i, j}^{n+1}-\widetilde{h}_{i, j}^{n}}{\Delta t}+\frac{\widetilde{F}_{i+1 / 2, j}^{n+1}-\widetilde{F}_{i-1 / 2, j}^{n+1}}{\Delta x}+\frac{\widetilde{F}_{i, j+1 / 2}^{n+1}-\widetilde{F}_{i, j-1 / 2}^{n+1}}{\Delta y}=0 .
$$

\subsubsection{Conservation law for sediments}

The approximation of the conservation law for the sediment height (14b) takes the same approach but is exposed here in reverse order. The interior scheme is given by

$$
\begin{aligned}
\frac{h_{i, j}^{n+1}-h_{i, j}^{n}}{\Delta t}+ & \frac{(\lambda F)_{i+1 / 2, j}^{n+1}-(\lambda F)_{i-1 / 2, j}^{n+1}}{\Delta x} \\
+ & \frac{(\lambda F)_{i, j+1 / 2}^{n+1}-(\lambda F)_{i, j-1 / 2}^{n+1}}{\Delta y}=0,
\end{aligned}
$$

where the reduced fluxes $\lambda F$ result from upwinding the limiter $\lambda$ with respect to the sign of $F=\mathbf{F} \cdot \mathbf{n}$. In other words,

$$
\begin{aligned}
& (\lambda F)_{i+1 / 2, j}^{n+1}=\lambda_{i, j}^{n+1}\left(F_{i+1 / 2, j}^{n+1}\right)^{+}+\lambda_{i+1, j}^{n+1}\left(F_{i+1 / 2, j}^{n+1}\right)^{-} \\
& (\lambda F)_{i, j+1 / 2}^{n+1}=\lambda_{i, j}^{n+1}\left(F_{i, j+1 / 2}^{n+1}\right)^{+}+\lambda_{i, j+1}^{n+1}\left(F_{i, j+1 / 2}^{n+1}\right)^{-} .
\end{aligned}
$$

Now, we need to define the $F$ 's. As in the previous case, let us recast the unconstrained sediment flux in a different way, i.e.,

$\mathbf{F}=\left(\widetilde{h}^{+}\right)^{3 / 2} \mathbf{G}-|\nabla h|^{p-2} \nabla \psi_{g}(h)$,

with

$\mathbf{G}=-|\nabla(\widetilde{h}+h)|^{1 / 2} \nabla \psi(h)$
Then, the discretization of $\mathbf{F} \cdot \mathbf{n}$ is carried out by upwinding $\left(\widetilde{h}^{+}\right)^{3 / 2}$ with respect to the sign of $G=\mathbf{G} \cdot \mathbf{n}$. For the second summand in the right-hand side of (33), we apply the same method as that employed for the $p$-Laplacian and the first conservation law. It follows that

$$
\begin{aligned}
F_{i+1 / 2, j}^{n+1}= & {\left[\left(\widetilde{h}_{i, j}^{n+1}\right)^{+}\right]^{3 / 2}\left(G_{i+1 / 2, j}^{n+1}\right)^{+}+\left[\left(\widetilde{h}_{i+1, j}^{n+1}\right)^{+}\right]^{3 / 2}\left(G_{i+1 / 2, j}^{n+1}\right)^{-} } \\
& -\frac{1}{2}\left[\left(B_{i+1 / 2, j-1 / 2}^{n}\right)^{p / 2-1}+\left(B_{i+1 / 2, j+1 / 2}^{n}\right)^{p / 2-1}\right] \\
& \times \frac{\psi_{g}\left(h_{i+1, j}^{n+1}\right)-\psi_{g}\left(h_{i, j}^{n+1}\right)}{\Delta x}, \quad(34 \mathrm{a}) \\
F_{i, j+1 / 2}^{n+1}=\left[\left(\widetilde{h}_{i, j}^{n+1}\right)^{+}\right]^{3 / 2}\left(G_{i, j+1 / 2}^{n+1}\right)^{+}+\left[\left(\widetilde{h}_{i, j+1}^{n+1}\right)^{+}\right]^{3 / 2}\left(G_{i, j+1 / 2}^{n+1}\right)^{-} & -\frac{1}{2}\left[\left(B_{i-1 / 2, j+1 / 2}^{n}\right)^{p / 2-1}+\left(B_{i+1 / 2, j+1 / 2}^{n}\right)^{p / 2-1}\right] \\
& \times \frac{\psi_{g}\left(h_{i, j+1}^{n+1}\right)-\psi_{g}\left(h_{i, j}^{n+1}\right)}{\Delta y},
\end{aligned}
$$

with

$$
\begin{array}{r}
G_{i+1 / 2, j}^{n+1}=-\frac{1}{2}\left[\left(\bar{B}_{i+1 / 2, j-1 / 2}^{n}\right)^{1 / 4}+\left(\bar{B}_{i+1 / 2, j+1 / 2}^{n}\right)^{1 / 4}\right] \\
\times \frac{\psi\left(h_{i+1, j}^{n+1}\right)-\psi\left(h_{i, j}^{n+1}\right)}{\Delta x}, \\
G_{i, j+1 / 2}^{n+1}=-\frac{1}{2}\left[\left(\bar{B}_{i-1 / 2, j+1 / 2}^{n}\right)^{1 / 4}+\left(\bar{B}_{i+1 / 2, j+1 / 2}^{n}\right)^{1 / 4}\right] \\
\times \frac{\psi\left(h_{i, j+1}^{n+1}\right)-\psi\left(h_{i, j}^{n+1}\right)}{\Delta y} .
\end{array}
$$

Note that no regularization is necessary in (35), as the exponent $1 / 4$ is positive.

\subsubsection{Complementarity equation}

To discretize the complementarity condition (14c), we first define the maximum erosion rate on each cell as

$E_{i, j}=\frac{1}{\Delta x \Delta y} \int_{\mathscr{C}_{i, j}} E(x, y) \mathrm{d} x \mathrm{~d} y$.

Then, using (32), we consider the discrete version

$$
\begin{aligned}
\min \left(1-\lambda_{i, j}^{n+1}, E_{i, j}-\frac{(\lambda F)_{i+1 / 2, j}^{n+1}-(\lambda F)_{i-1 / 2, j}^{n+1}}{\Delta x}\right. & \\
\left.-\frac{(\lambda F)_{i, j+1 / 2}^{n+1}-(\lambda F)_{i, j-1 / 2}^{n+1}}{\Delta y}\right) & =0
\end{aligned}
$$

of $(14 \mathrm{c})$. Let us transform this equation into a more valuable form, for theoretical as well as numerical purposes, by introducing two notions. The first one represents the total unlimited outgoing flux from cell $\mathscr{C}_{i, j}$, i.e.,

$$
\begin{aligned}
\langle F\rangle_{i, j}^{n+1} & =\Delta y\left[\left(F_{i+1 / 2, j}^{n+1}\right)^{+}+\left(F_{i-1 / 2, j}^{n+1}\right)^{-}\right] \\
& +\Delta x\left[\left(F_{i, j+1 / 2}^{n+1}\right)^{+}+\left(F_{i, j-1 / 2}^{n+1}\right)^{-}\right],
\end{aligned}
$$


while the second one represents the total limited incoming flux from cell $\mathscr{C}_{i, j}$, i.e.,

$$
\begin{aligned}
\backslash \lambda F\left\langle_{i, j}^{n+1}\right. & =\Delta y\left[\lambda_{i-1, j}^{n+1}\left(F_{i-1 / 2, j}^{n+1}\right)^{+}+\lambda_{i+1, j}^{n+1}\left(F_{i+1 / 2, j}^{n+1}\right)^{-}\right] \\
& +\Delta x\left[\lambda_{i, j-1}^{n+1}\left(F_{i, j-1 / 2}^{n+1}\right)^{+}+\lambda_{i, j+1}^{n+1}\left(F_{i, j+1 / 2}^{n+1}\right)^{-}\right] .
\end{aligned}
$$

Then, equation (37) receives the more compact form

$\min \left(1-\lambda_{i, j}^{n+1}, E_{i, j}-\lambda_{i, j}^{n+1} \frac{\langle F\rangle_{i, j}^{n+1}}{\Delta x \Delta y}+\frac{\rangle \lambda F\left\langle_{i, j}^{n+1}\right.}{\Delta x \Delta y}\right)=0$,

in which $\lambda_{i, j}^{n+1}$ in the second argument has been isolated from the neighboring $\lambda^{n+1}$ 's. The idea is now to calibrate this new relation so that the coefficient of $\lambda_{i, j}^{n+1}$ in the second argument is -1 . To this end, we invoke the equivalence property

$\min (a, b)=0 \Longleftrightarrow \min (a, \gamma b)=0, \quad \forall \gamma>0$.

In the "good" case $\langle F\rangle_{i, j}^{n+1} \neq 0$, by setting $\gamma=\Delta x \Delta y /\langle F\rangle_{i, j}^{n+1}$, equation (39) becomes

$\min \left(1-\lambda_{i, j}^{n+1}, \frac{\left.\Delta x \Delta y E_{i, j}+\right\rangle \lambda F\left\langle_{i, j}^{n+1}\right.}{\langle F\rangle_{i, j}^{n+1}}-\lambda_{i, j}^{n+1}\right)=0$.

By virtue of $\min (a-c, b-c)=\min (a, b)-c$, we can pull $\lambda_{i, j}^{n+1}$ out of the min and obtain

$\lambda_{i, j}^{n+1}=\min \left(1, \frac{\left.\Delta x \Delta y E_{i, j}+\right\rangle \lambda F\left\langle_{i, j}^{n+1}\right.}{\langle F\rangle_{i, j}^{n+1}}\right)$.

In the "bad" case $\langle F\rangle_{i, j}^{n+1}=0$, the relation (39) reduces to

$\min \left(1-\lambda_{i, j}^{n+1}, E_{i, j}+\frac{\rangle \lambda F\left\langle_{i, j}^{n+1}\right.}{\Delta x \Delta y}\right)=0$.

Owing to the definition of various terms, we see that the second argument of the min is positive (see also the Lemma 4). Then the only way to solve this equation is to have $\lambda_{i, j}^{n+1}=1$. This result should not come as a surprise. Indeed, having $\langle F\rangle_{i, j}^{n+1}=0$ means there is no outgoing fluxes from the cell $\mathscr{C}_{i, j}$, that is to say, erosion does not occur. Thus, there is no need to limit the sediment flux to satisfy the constraint on the erosion process, whence $\lambda_{i, j}^{n+1}=1$.

Anticipating the boundary conditions that will be addressed in $\$ 4.3 .4$, we wish to point out one important issue regarding the numerical resolution. Contrary to Gervais and her coauthors $[13,14,20-22]$, we advocate mounting the whole set of equations (30)-(36), (40)-(46) into a single system in the unknowns $\left(\widetilde{h}_{i, j}^{n+1}, h_{i, j}^{n+1}, \lambda_{i, j}^{n+1}\right)$ and applying Newton's method to this system. In [13,14,20-22], the strategy for numerical resolution rested on a variable-switching procedure whereby, as examplified in Eymard et al. [17, $\S 7.2 .3]$ for a compositional multiphase flow problem, the set of equations and the set of unknowns evolve locally and dynamically with the iterates, according to the presumed flow regime (i.e., whether or not the erosion constraint is thought to be saturated). The main advantage of our strategy is to make implementation much easier by providing a unified formulation to the problem at the discrete level.

It could be argued that the min function is not differentiable everywhere, which could cause trouble to Newton's method. In fact, we recommend the Newton-min method, a variant in which any element of the subdifferential can be selected in place of the corresponding partial derivative. The Newton-min method has been proved to work well by many authors (see, e.g., Ben Gharbia [5]).

\subsubsection{Boundary conditions and initial data}

The boundary conditions are discretized via an approach similar to what was done for the $p$-Laplacian case. For each ghost cell, two new equations are considered to take into account the water and sediment inflows. Moreover, on these cells the flux limiter $\lambda$ is set to the value 1 , which amounts to saying that the prescribed boundary conditions are never truncated. For the water fluxes, the equations are

$$
\begin{aligned}
& \Gamma_{b}: \widetilde{F}_{i, 1 / 2}^{n+1}+\frac{1}{\Delta x \Delta t} \int_{t^{n}}^{t^{n+1}} \mathrm{~d} t \int_{(i-1) \Delta x}^{i \Delta x} \widetilde{\phi}(x, 0, t) \mathrm{d} x=0, \\
& \Gamma_{l}: \widetilde{F}_{1 / 2, j}^{n+1} \quad+\frac{1}{\Delta y \Delta t} \int_{t^{n}}^{t^{n+1}} \mathrm{~d} t \int_{(j-1) \Delta y}^{j \Delta y} \widetilde{\phi}(0, y, t) \mathrm{d} y=0, \\
& \Gamma_{t}: \widetilde{F}_{i, N_{y}+1 / 2}^{n+1}-\frac{1}{\Delta x \Delta t} \int_{t^{n}}^{t^{n+1}} \mathrm{~d} t \int_{(i-1) \Delta x}^{i \Delta x} \widetilde{\phi}\left(x, L_{y}, t\right) \mathrm{d} x=0, \\
& \Gamma_{r}: \widetilde{F}_{N_{x}+1 / 2, j}^{n+1}-\frac{1}{\Delta y \Delta t} \int_{t^{n}}^{t^{n+1}} \mathrm{~d} t \int_{(j-1) \Delta y}^{j \Delta y} \widetilde{\phi}\left(L_{x}, y, t\right) \mathrm{d} y=0,
\end{aligned}
$$

and for the sediment fluxes, the equations are

$$
\begin{aligned}
& \Gamma_{b}: F_{i, 1 / 2}^{n+1}+\frac{1}{\Delta x \Delta t} \int_{t^{n}}^{t^{n+1}} \mathrm{~d} t \int_{(i-1) \Delta x}^{i \Delta x} \phi(x, 0, t) \mathrm{d} x=0, \\
& \Gamma_{l}: F_{1 / 2, j}^{n+1}+\frac{1}{\Delta y \Delta t} \int_{t^{n}}^{t^{n+1}} \mathrm{~d} t \int_{(j-1) \Delta y}^{j \Delta y} \phi(0, y, t) \mathrm{d} y=0, \\
& \Gamma_{t}: F_{i, N_{y}+1 / 2}^{n+1}-\frac{1}{\Delta x \Delta t} \int_{t^{n}}^{t^{n+1}} \mathrm{~d} t \int_{(i-1) \Delta x}^{i \Delta x} \phi\left(x, L_{y}, t\right) \mathrm{d} x=0, \\
& \Gamma_{r}: F_{N_{x}+1 / 2, j}^{n+1}-\frac{1}{\Delta y \Delta t} \int_{t^{n}}^{t^{n+1}} \mathrm{~d} t \int_{(j-1) \Delta y}^{j \Delta y} \phi\left(L_{x}, y, t\right) \mathrm{d} y=0 .
\end{aligned}
$$

As far as the corner ghost cells are concerned, we consider two new equations that are extrapolation of the water and sediment heights from the neighbouring cells. For the 
water height, these are

$$
\begin{array}{rllll}
\mathscr{C}_{0,0} & : \widetilde{h}_{0,0}^{n+1}+\widetilde{h}_{1,1}^{n+1}-\widetilde{h}_{1,0}^{n+1}-\widetilde{h}_{0,1}^{n+1}=0, \\
\mathscr{C}_{N_{x}+1,0}: & : \widetilde{h}_{N_{x}+1,0}^{n+1}+\widetilde{h}_{N_{x}, 1}^{n+1}-\widetilde{h}_{N_{x}+1,1}^{n+1}-\widetilde{h}_{N_{x}, 0}^{n+1}=0, \\
\mathscr{C}_{0, N_{y}+1}: & : \widetilde{h}_{0, N_{y}+1}^{n+1}+\widetilde{h}_{1, N_{y}}^{n+1}-\widetilde{h}_{1, N_{y}+1}^{n+1}-\widetilde{h}_{0, N_{y}}^{n+1}=0, \\
\mathscr{C}_{N_{x}+1, N_{y}+1}: & : \widetilde{h}_{N_{x}+1, N_{y}+1}^{n+1}+\widetilde{h}_{N_{x}, N_{y}}^{n+1}-\widetilde{h}_{N_{x}, N_{y}+1}^{n+1}-\widetilde{h}_{N_{x}+1, N_{y}}^{n+1}=0 .
\end{array}
$$

For the sediment height, these are

$$
\begin{array}{rlrl}
\mathscr{C}_{0,0}: & h_{0,0}^{n+1}+h_{1,1}^{n+1}-h_{1,0}^{n+1}-h_{0,1}^{n+1}=0, \\
\mathscr{C}_{N_{x}+1,0}: h_{N_{x}+1,0}^{n+1}+h_{N_{x}, 1}^{n+1}-h_{N_{x}+1,1}^{n+1}-h_{N_{x}, 0}^{n+1}=0, \\
\mathscr{C}_{0, N_{y}+1}: h_{0, N_{y}+1}^{n+1}+h_{1, N_{y}}^{n+1}-h_{1, N_{y}+1}^{n+1}-h_{0, N_{y}}^{n+1}=0, \\
\mathscr{C}_{N_{x}+1, N_{y}+1}: h_{N_{x}+1, N_{y}+1}^{n+1}+h_{N_{x}, N_{y}}^{n+1}-h_{N_{x}, N_{y}+1}^{n+1}-h_{N_{x}+1, N_{y}}^{n+1}=0 .
\end{array}
$$

We conclude this section by detailing the discretization of the initial data $\widetilde{h}^{0}$ and $h^{0}$. In the domain $\Omega$, these are chosen as piecewise-constant functions whose cell values are

$\widetilde{h}_{i, j}^{0}=\frac{1}{\Delta x \Delta y} \int_{\mathscr{C}_{i, j}} \widetilde{h}^{0}(x, y) \mathrm{d} x \mathrm{~d} y$,

$h_{i, j}^{0}=\frac{1}{\Delta x \Delta y} \int_{\mathscr{C}_{i, j}} h^{0}(x, y) \mathrm{d} x \mathrm{~d} y$.

Regarding the values in the ghost cells, for the sake of simplicity we choose to use an extrapolation of the values computed in (46). We can also determine an initial value for the flux limiter, which is in practice only used as an initial guess for the first Newton iteration. To do so, one method consists in: (i) sorting cells in decreasing topography order, and (ii) applying formula (40) while visiting the cells in this specific order. This method, introduced by Granjeon [24] in a more intuitive language, avoids the resolution of a linear system.

\section{Properties of the numerical scheme}

The numerical scheme (30)-(36), (40)-(46) enjoys many favorable properties that deserve mentioning. The first property is an estimate on the flux limiter $\lambda$.

Lemma 4 (Estimates on the flux limiter) For all $n \geqslant 0$, for all $1 \leqslant i \leqslant N_{x}, 1 \leqslant j \leqslant N_{y}$, we have

$0<\lambda_{i, j}^{n+1} \leqslant 1$.

Proof In formula (40), we observe that $\Delta x \Delta y E_{i, j}>0$ and $\langle F\rangle_{i, j}^{n+1} \geqslant 0$. We thus have to show that $\rangle \lambda F\left\langle_{i, j}^{n+1} \geqslant 0\right.$.

Let us suppose that cells are sorted in decreasing topographical order. Then, computing the discrete flux limiter in this specific order, we can see that the term $\rangle \lambda F\left\langle\begin{array}{l}n+1 \\ i, j\end{array}\right.$ involves unknowns $\lambda_{i, j}^{n+1}$ computed only in previous cells.
In particular, for the one with the highest sediment height, \rangle$\lambda F\left\langle_{i, j}^{n+1}=0\right.$ (or $\rangle \lambda F\left\langle_{i, j}^{n+1}>0\right.$ if it is located near the boundary where inflow of sediments is prescribed). By propagating positivity from the highest cell to lower ones, every cell can be reached. This completes the proof.

The next statement is the discrete of Lemmas 1 and 2 .

Lemma 5 (Estimates on water and sediment heights) For all $n \geqslant 0$, for all $1 \leqslant i \leqslant N_{x}, 1 \leqslant j \leqslant N_{y}$, we have

$\widetilde{h}_{i, j}^{n} \geqslant 0$,

$h_{i, j}^{n} \geqslant h_{*}$.

Proof The first estimate is proven by induction. Suppose that (48a) holds at $n$ and assume that $\widetilde{h}_{i^{*}, j^{*}}^{n+1}<0$ for some $\left(i^{*}, j^{*}\right)$. Then, analyzing the sign of the fluxes associated to $\mathscr{C}_{i^{*}, j^{*}}$ and applying the update formula (30), it turns out that the latter leads to $\widetilde{h}_{i^{*}, j^{*}}^{n+1} \geqslant 0$, which is a contradiction.

The second estimate also relies on induction. Suppose that (48b) holds at $n$ and let $h_{i^{*}, j^{*}}^{n+1}=\min _{i, j} h_{i, j}^{n+1}$. As before, the claim is proven by applying the numerical scheme (31) and studying the sign of the corresponding fluxes (note that $\psi$ and $\psi_{g}$ are increasing functions).

Lemma 3 also has the following discrete counterpart.

Lemma 6 (Mass conservation of water and sediments) For all $n \geqslant 0$, one has

$$
\begin{aligned}
& \Delta x \Delta y \sum_{i=1}^{N_{x}} \sum_{j=1}^{N_{y}} \widetilde{h}_{i, j}^{n}=\int_{\Omega} \widetilde{h}_{0}(x, y) \mathrm{d} x \mathrm{~d} y-\int_{0}^{t^{n}} \int_{\partial \Omega} \widetilde{\phi}(x, y, t) \mathrm{d} \sigma \mathrm{d} t, \\
& \Delta x \Delta y \sum_{i=1}^{N_{x}} \sum_{j=1}^{N_{y}} h_{i, j}^{n}=\int_{\Omega} h_{0}(x, y) \mathrm{d} x \mathrm{~d} y-\int_{0}^{t^{n}} \int_{\partial \Omega} \phi(x, y, t) \mathrm{d} \sigma \mathrm{d} t .
\end{aligned}
$$

Proof Again, we proceed by induction. For $n=0$, this is due to the discretization of the initial data (46). If (49) holds at $n$, then by adding the first equation with the sum of (30) over $(i, j)$, by adding the second equation with the sum of (31)) over $(i, j)$, by using the conservativity of the scheme and the definition of the boundary fluxes (42)-(43), we get the result.

The last property we wish to put forward is the existence of a discrete solution. Far from being trivail, this property is of practical importance for the Newton method to have a chance to converge.

Proposition 1 (Existence of a discrete solution) Let $\widetilde{h}_{i, j}^{n}$ and $h_{i, j}^{n}$ be such that (48)-(49) hold. Then, for all $\Delta t_{n}>0$, there exists at least one solution $\left(\widetilde{h}_{i, j}^{n+1}, h_{i, j}^{n+1}, \lambda_{i, j}^{n+1}\right)_{i, j}$ to the numerical scheme (30)-(36), (40)-(46), satisfying the properties (47)-(49). 
Proof The result follows from a topological degree argument that we only sketch out, referring the readers to the standard literature $[11,15,27]$ or to Peton [28] for full details. The basic idea is to define a homotopy $\mathscr{H}_{\mu}, \mu \in[0,1]$ between our system of equations and a simpler one, for which the existence of a unique solution is trivial. The difficulty lies in making sure that some technical conditions are satisfied along the homotopy.

In our problem, let us consider the system

$$
\begin{aligned}
\frac{\widetilde{h}_{i, j}^{\mu, n+1}-\widetilde{h}_{i, j}^{n}}{\Delta t}+\mu\left[\frac{\widetilde{F}_{i+1 / 2, j}^{\mu, n+1}-\widetilde{F}_{i-1 / 2, j}^{\mu, n+1}}{\Delta x}+\frac{\widetilde{F}_{i, j+1 / 2}^{\mu, n+1}-\widetilde{F}_{i, j-1 / 2}^{\mu, n+1}}{\Delta y}\right] & =0, \\
\frac{h_{i, j}^{\mu, n+1}-h_{i, j}^{n}}{\Delta t}+\mu\left[\frac{F_{i+1 / 2, j}^{\mu, n+1}-F_{i-1 / 2, j}^{\mu, n+1}}{\Delta x}+\frac{F_{i, j+1 / 2}^{\mu, n+1}-F_{i, j-1 / 2}^{\mu, n+1}}{\Delta y}\right] & =0, \\
\lambda_{i, j}^{\mu, n+1}+\mu-1-\mu \min \left(1, \frac{\left.\Delta x \Delta y E_{i, j}+\right\rangle \lambda F F_{i, j}^{\mu, n+1}}{\langle F\rangle_{i, j}^{\mu, n+1}}\right) & =0 .
\end{aligned}
$$

For $\mu=1$ we recover the numerical scheme (30)-(36), (40)(46), while taking $\mu=0$ leads to a simple mapping for which the topological degree is equal to 1 . What is left to show is that the variables $\left(\widetilde{h}_{i, j}^{\mu, n+1}, h_{i, j}^{\mu, n+1}, \lambda_{i, j}^{\mu, n+1}\right)$ remain bounded for all $\mu \in[0,1]$. This can be achieved by adapting the proofs of the estimates (47)-(49). Thus all the mappings $\mathscr{H}_{\mu}, \mu \in$ $[0,1]$, have a topological degree equal to 1 , which implies the existence of at least one solution.

\section{Numerical results}

In this section, we present two test cases in order to illustrate the stratigraphic model as well as the capabilities of the numerical scheme.

\subsection{Delta evolution}

This first configuration aims to highlight the impact of water effects in the stratigraphic model. Let us consider a domain of size $20 \mathrm{~km} \times 20 \mathrm{~km}$ divided into marine and continental parts, the sea level being fixed at $H_{m}=1.4 \mathrm{~km}$. Table 1 gathers the values of the diffusion coefficients. The gravity ones are intended to be small in order to focus on bed load transport. Concerning $\widetilde{K}$, its value is set to $20000 \mathrm{~km}^{\frac{1}{2}} / \mathrm{My}$. We also take into account a constraint on the erosion process through the maximum weathering rate $E=0.1 \mathrm{~km} / \mathrm{My}$. Inflows of water and sediments are prescribed at the boundary as

$$
-\int_{\partial \Omega} \widetilde{\phi} \mathrm{d} \sigma=40 \mathrm{~km}^{3} / \mathrm{My}, \quad-\int_{\partial \Omega} \phi \mathrm{d} \sigma=0.04 \mathrm{~km}^{3} / \mathrm{My},
$$

for all $t \geqslant 0$.

The computational domain is divided into $200 \times 200$ cells, and the evolution of the system is simulated over $1 \mathrm{My}$.

\begin{tabular}{ccc}
\hline \multirow{2}{*}{ Coefficient } & \multicolumn{2}{c}{ Domain } \\
\cline { 2 - 3 } & Marine & Continental \\
\hline$K_{g}\left(\mathrm{~km}^{2} / \mathrm{My}\right)$ & 0.01 & 0.04 \\
$K\left(\mathrm{~km}^{1 / 2} / \mathrm{My}\right)$ & 3000 & 10000 \\
\hline
\end{tabular}

Table 1: Diffusion coefficients value.

The value of the exponent of the $p$-Laplacian in the sediment flux (15b) is set to $p=2.5$. We start the simulation with the initial time-step $\Delta t^{0}=10^{-4} \mathrm{My}$. At each time-step, the nonlinear system is solved using Newton's method, at a precision of $10^{-5} \mathrm{~km}$ for the water and sediment heights, and $10^{-5}$ for the flux limiter (based on the infinity norm of the residual). The resolution of the linear systems arising at each Newton iteration is made using the BiCGStab [31] method from PETSc routines [3]. If the time iteration is accepted, the next time-step is defined as $\Delta t^{n+1}=1.1 \Delta t^{n}$, up to the maximum value $10^{-3} \mathrm{My}$. Otherwise, if Newton's method fails to converge after 20 iterations, the time-step is rejected and is restarted with $\Delta t^{n}=0.5 \Delta t^{n}$.

Figure 6 illustrates the behaviour of the system. Because of the low gravity diffusion coefficients, the sediment transport is mainly due to water fluxes. By looking at Figure 6c, we observe that water tends to carry away sediments from the continental part. Moreover, the associated transport being strong, the sediment flux needs to be limited (see the evolution of the flux limiter) in order to satisfy the constraint on the erosion rate. As time goes by, we can also distinguish the formation of a delta near the shoreline. In particular, we observe a break between the continental and marine domains, which is due to the discontinuity within the diffusion coefficients.

In Table 2 are gathered some numerical data, obtained at the end of the simulation. We show the importance of choosing a good preconditioner to solve the underlying system properly. Among the configurations tested, we have always a low number of refused time-steps. Nevertheless, we observe that the preconditioner choice leads to important changes in the computational time. In our case, it is better to use ILU(2) or ILU(3). For stronger preconditioning methods, the cost of each iteration becomes too important to gain in efficiency. On the opposite situation, ILU(0) turns out to not be a good choice because of the high number of iterations needed at each Newton step.

\subsection{Lakes formation}

In this second configuration, we aim to reproduce the filling of lakes. Once again, we consider a domain of size $20 \mathrm{~km} \times$ $20 \mathrm{~km}$ divided in marine and continental regions. The sea level separating these two parts is set to $H_{m}=1.8 \mathrm{~km}$. The 


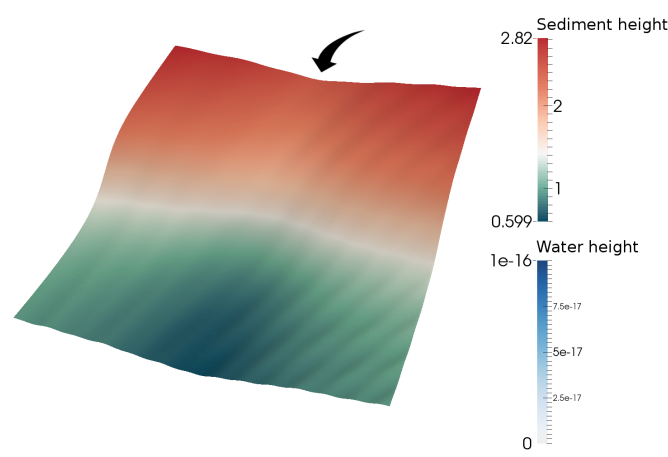

(a) Initial water and sediment heights $(\mathrm{km})$.

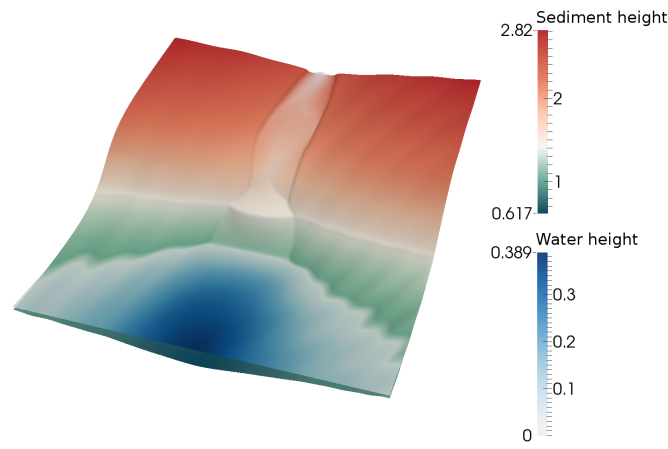

(c) Water and sediment heights (km) at time $T=0.5 \mathrm{My}$.

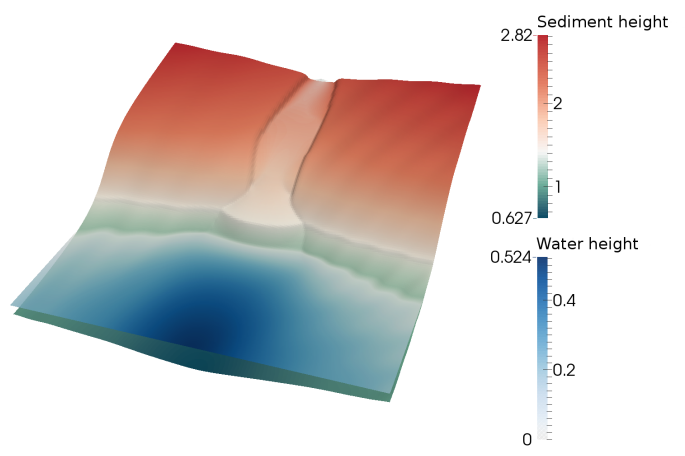

(e) Water and sediment heights $(\mathrm{km})$ at time $T=1 \mathrm{My}$.

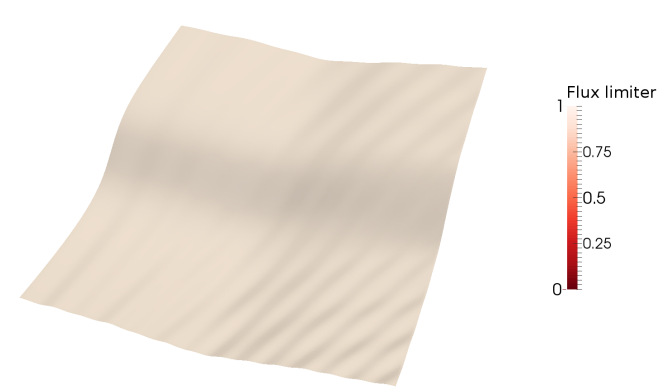

(b) Initial flux limiter.

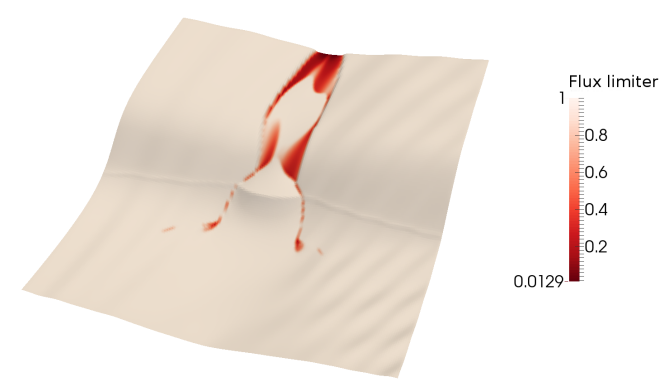

(d) Flux limiter at time $T=0.5 \mathrm{My}$.

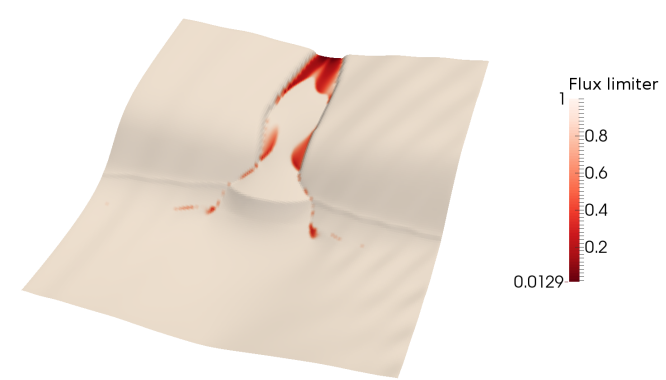

(f) Flux limiter at time $T=1 \mathrm{My}$.

Fig. 6: Evolution of the water and sediment heights and of the flux limiter.

\begin{tabular}{lccccc}
\hline \multirow{2}{*}{ Numerical data } & \multicolumn{5}{c}{ Preconditioner } \\
\cline { 2 - 6 } & ILU(0) & ILU(1) & ILU(2) & ILU(3) & ILU(4) \\
\hline Accepted time-steps & 1117 & 1117 & 1117 & 1117 & 1117 \\
Refused time-steps & 40 & 40 & 40 & 40 & 40 \\
Mean Newton iterations per time-step & 5.92 & 5.12 & 4.85 & 4.55 & 4.33 \\
Mean solver iterations per Newton iteration & 56.32 & 34.93 & 27.65 & 19.96 & 15.93 \\
CPU time (s) & 4521 & 3456 & 3286 & 3277 & 3631 \\
\hline
\end{tabular}

Table 2: Numerical results. 


\begin{tabular}{ccc}
\hline \multirow{2}{*}{ Coefficient } & \multicolumn{2}{c}{ Domain } \\
\cline { 2 - 3 } & Marine & Continental \\
\hline$K_{g}\left(\mathrm{~km}^{2} / \mathrm{My}\right)$ & 40 & 5 \\
$K\left(\mathrm{~km}^{1 / 2} / \mathrm{My}\right)$ & 6000 & 3000 \\
\hline
\end{tabular}

Table 3: Diffusion coefficients value.

value of the diffusion coefficients is given in Table 3 , and $\widetilde{K}$ is fixed to $10000 \mathrm{~km}^{1 / 2} / \mathrm{My}$. A constraint on the erosion process is taken into account with the maximum weathering rate $E=0.1 \mathrm{~km} / \mathrm{My}$. Finally, inflows of water and sediments are prescribed at the boundary, which satisfy

$-\int_{\partial \Omega} \widetilde{\phi} \mathrm{d} \sigma=46 \mathrm{~km}^{3} / \mathrm{My}, \quad-\int_{\partial \Omega} \phi \mathrm{d} \sigma=0.046 \mathrm{~km}^{3} / \mathrm{My}$,

for all $t \geqslant 0$.

Concerning the numerical parameters: first, the domain is approximated by a regular grid of $200 \times 200$ cells. The evolution of the system is simulated over $1 \mathrm{My}$. The exponent of the $p$-Laplacian in the sediment flux (15b) is fixed to $p=2.5$. For the stopping criteria of Newton's method and the management of the time-step, we choose the same methodology as for the first test case.

The evolution of the system is presented in Figure 7. In a first phase, the water inflows fill the upper part of the domain with the creation of lakes (Figure 7c). Then, once the reservoir capacity is reached, water flows downhill carrying away sediments from the central area (Figure 7e). Furthermore, on the pictures displaying the evolution of the flux limiter, one can see that the main areas where the sediment flux needs to be limited (in red) correspond to mountain flanks (where the slope $\nabla h$ is high). From time $T=0.5 \mathrm{My}$, a new constrained zone appears in the central zone of the domain. This is due to the water flowing to the lower part, which tends to transport a large quantity of sediments. The sediment flux thus has to be limited to respect the constraint on the weathering rate.

In Table 4 are presented some numerical data. As in the previous test case, we observe that the choice of preconditioner has a tremendous impact on the computational time. Among the configurations tested, ILU(2) and ILU(3) still seem to be the better ones.

\section{Extension to the multi-lithology case}

Gervais and Masson [20, 22] went on developing a multilithology version of their initial model in order to capture effects due to the simultaneous presence of various types of sediments. The purpose of this section is to demonstrate that our stratigraphic model (14)-(18) is also extendable to the multi-lithology case.

\subsection{Physical model}

In geology, sediments are classified according to different criteria, e.g., the mineral composition, grain size... Among these categories, called lithologies, we can find sands and shales. Each of these may have its own physical properties, such as the transportability (i.e., the diffusion speed) which will be our main concern in this section.

The notations used in the sequel are presented in Figure 8. We suppose sediments are made up of a mixture of $L$ lithologies, the concentrations $\left(c_{\ell}\right)_{\ell=1, \ldots, L} \in[0,1]^{L}$ of which naturally satisfy the relation

$\sum_{\ell=1}^{L} c_{\ell}=1$.

Instead of considering one conservation law on the sediment height as before, the idea is to seek a balance law for each fraction of lithology. This notion is computed over the sediment thickness on each point of the domain, that is, as a function of the depth $\zeta \in\left[h_{*}, h(x, y, t)\right]$, where $h_{*}$ is the minimum sediment height. More precisely, it is defined through the formula

$\mathfrak{F}_{\ell}(x, y, t)=\int_{h_{*}}^{h(x, y, t)} c_{\ell}(x, y, \zeta, t) \mathrm{d} \zeta, \quad \ell=1, \ldots, L$.

The corresponding time derivative is given by

$\partial_{t} \mathfrak{F}_{\ell}=\left.c_{\ell}\right|_{\zeta=h} \partial_{t} h+\int_{h_{*}}^{h} \partial_{t} c_{\ell} \mathrm{d} \zeta$.

We now have to set the corresponding lithology flux. To do so, we follow the procedures of Gervais [20] and Rivenæs [30]. The first step is to extend the diffusion coefficients $K_{g}$ and $K$ of the previous model to the case of multiple lithologies. For $\ell=1, \ldots, L$, we define $K_{g, \ell}$ and $K_{\ell}$ by

$$
\begin{gathered}
K_{g, \ell}(h)= \begin{cases}K_{g, \ell, m}, & \text { if } h<H_{m}, \\
K_{g, \ell, c}, & \text { if } h \geqslant H_{m},\end{cases} \\
K_{\ell}(h)= \begin{cases}K_{\ell, m}, & \text { if } h<H_{m}, \\
K_{\ell, c}, & \text { if } h \geqslant H_{m} .\end{cases}
\end{gathered}
$$

Next, we introduce a new type of concentration, named surface concentrations and denoted by $\left(c_{\ell}^{s}\right)_{\ell=1, \ldots, L} \in[0,1]^{L}$. These are defined only at the surface of the sedimentary basin, and aimed at representing the sediment transport which occurs nowhere but at the surface. Moreover, they satisfy the property

$$
\sum_{\ell=1}^{L} c_{\ell}^{s}=1 .
$$

As a consequence, concentrations within the basin do not change over time, i.e.,

$\partial_{t} c_{\ell}=0, \quad$ in $\Omega \times\left[h_{*}, h\left[\times \mathbb{R}_{+}, \quad \ell=1, \ldots, L\right.\right.$. 


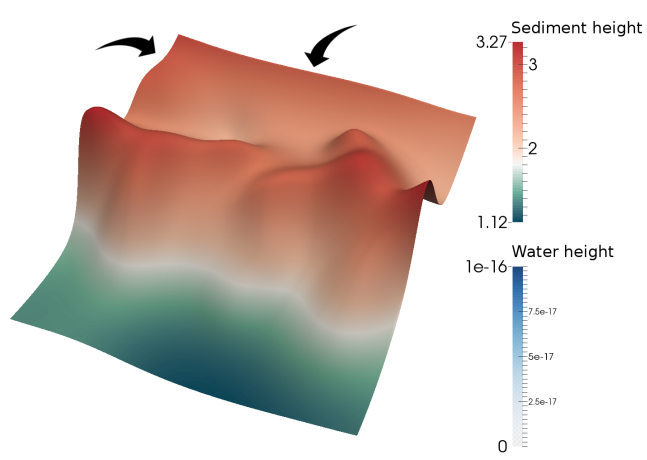

(a) Initial water and sediment heights $(\mathrm{km})$.

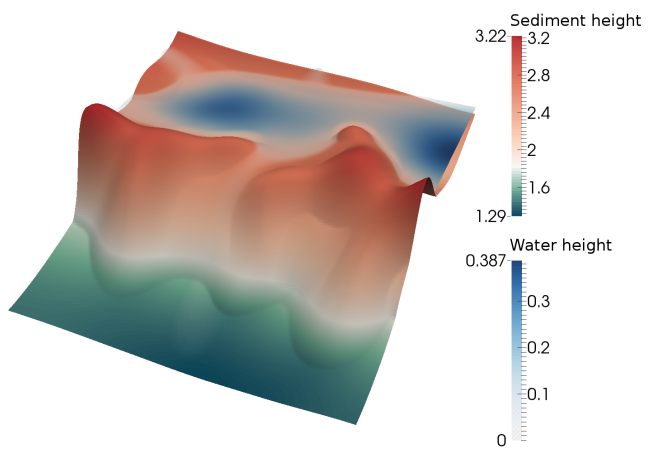

(c) Water and sediment heights (km) at time $T=0.5 \mathrm{My}$.

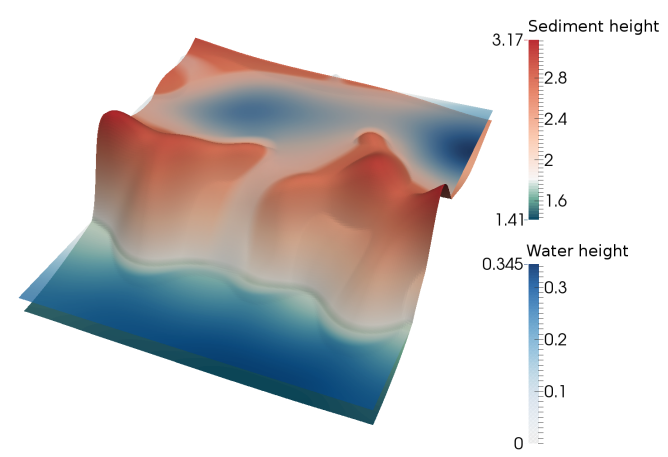

(e) Water and sediment heights $(\mathrm{km})$ at time $T=1 \mathrm{My}$.

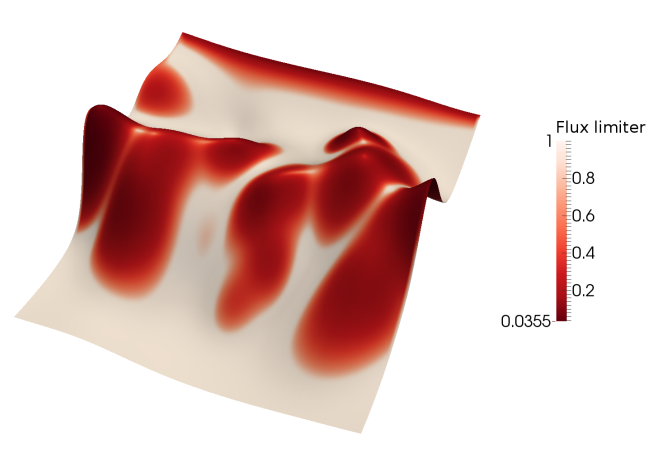

(b) Initial flux limiter.

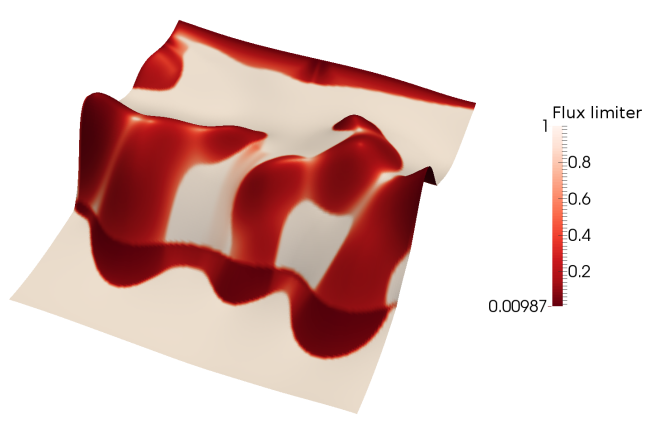

(d) Flux limiter at time $T=0.5 \mathrm{My}$.

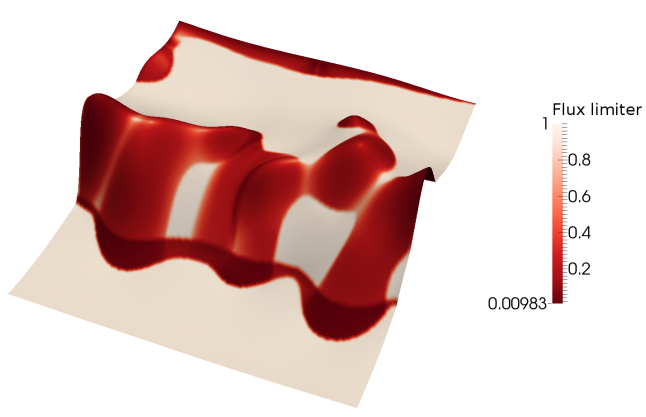

(f) Flux limiter at time $T=1 \mathrm{My}$.

Fig. 7: Evolution of the water and sediment heights and of the flux limiter.

\begin{tabular}{lccccc}
\hline \multirow{2}{*}{ Numerical data } & \multicolumn{5}{c}{ Preconditioner } \\
\cline { 2 - 6 } & ILU(0) & ILU(1) & ILU(2) & ILU(3) & ILU(4) \\
\hline Accepted time-steps & 1077 & 1077 & 1077 & 1077 & 1075 \\
Refused time-steps & 26 & 26 & 26 & 26 & 26 \\
Mean Newton iterations per time-step & 5.53 & 4.88 & 4.59 & 4.40 & 4.23 \\
Mean solver iterations per Newton iteration & 92.39 & 57.15 & 45.08 & 32.89 & 26.05 \\
CPU time (s) & 6026 & 4519 & 4034 & 4017 & 4203 \\
\hline
\end{tabular}

Table 4: Numerical results. 


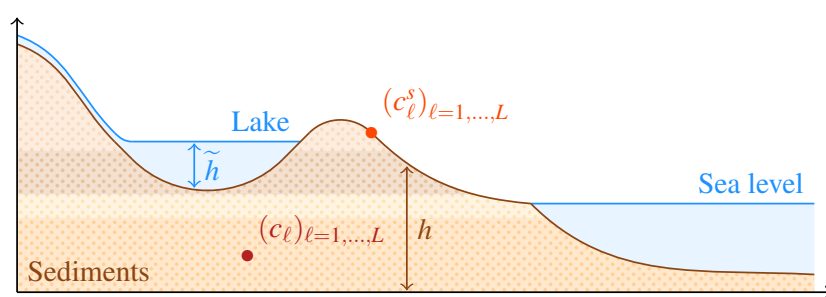

Fig. 8: Notations for the stratigraphic model: $\tilde{h}$ denotes the water height, $h$ the sediment height, $c_{\ell}$ the concentration of lithology $\ell$ and $c_{\ell}^{s}$ the surface concentration of lithology $\ell$.

These can change only during sedimentation, where the composition of deposited sediments is given by the surface concentrations, that is to say, if $\partial_{t} h>0$ then

$\left.c_{\ell}\right|_{\zeta=h}=c_{\ell}^{s}, \quad$ in $\Omega \times \mathbb{R}_{+}, \quad \ell=1, \ldots, L$.

From this, the time derivative of the fraction of lithology $\ell$ (52) can be written in the simpler form

$\partial_{t} \mathfrak{F}_{\ell}=\left.c_{\ell}\right|_{\zeta=h} \partial_{t} h$.

Then, the conservation law for each fraction of lithology $\ell=$ $1, \ldots, L$ is taken to be

$\left.c_{\ell}\right|_{\zeta=h} \partial_{t} h+\operatorname{div}\left(\lambda \mathbf{F}_{\ell}\right)=0, \quad$ in $\Omega \times \mathbb{R}_{+}$,

with the corresponding lithology flux

$$
\begin{aligned}
\mathbf{F}_{\ell}=-K_{\ell}(h) c_{\ell}^{s}\left(\widetilde{h}^{+}\right)^{3 / 2}|\nabla(\widetilde{h}+h)|^{1 / 2} \nabla h & \\
& -K_{g, \ell}(h) c_{\ell}^{s}|\nabla h|^{p-2} \nabla h .
\end{aligned}
$$

To take into account the constraint on the erosion process, we apply a procedure similar to that of (13) for the single-lithology model. Summing the equations (58) over $\ell=1, \ldots, L$ gives

$\partial_{t} h+\sum_{\ell=1}^{L} \operatorname{div}\left(\lambda \mathbf{F}_{\ell}\right)=0$.

It is therefore natural to impose the complementarity condition

$\min \left(1-\lambda, E-\sum_{\ell=1}^{L} \operatorname{div}\left(\lambda \mathbf{F}_{\ell}\right)\right)=0, \quad$ in $\Omega \times \mathbb{R}_{+}$.

The balance law for the water height is exactly the same equation as (14a). Thus, the multilithological model includes the conservation laws (14a) and (58), the complementarity condition (60) and the closure equation (54). The associated unknowns are the water and sediment heights $\widetilde{h}$ and $h$, the flux limiter $\lambda$, and the surface concentrations $\left(c_{\ell}^{s}\right)_{\ell=1, \ldots, L}$. As for the boundary conditions, we consider inflow of water and lithologies.

In the above multi-lithology model, some geologically realistic processes such as changing sea level, subsidence and compaction have been omitted in order to simplify the exposition. There is no difficutly in taking these into account, except for the fact that the become equations longer and the presentation heavier. In $\$ 7.3 .2$, we will show a test case with eustasy using a given history for the sea level. Needless to say, these additional processes are fully incorporated into the DionisosFlow software.

\subsection{Finite Volume discretization}

In the discretization of the equations, a new difficulty emerges regarding the approximation of sediment columns. Indeed, it is now essential to determine the proportion of each type of sediment during the erosion and sedimentation processes. Let $\zeta$ be the coordinate in the vertical direction. Following Gervais [20], the concentration $c_{\ell, i, j}^{n+1}(\zeta)$ is defined as the solution at time $t^{n+1}$ of the system

$$
\begin{array}{lll}
\partial_{t} c_{\ell, i, j}(\zeta, t)=0, & \zeta \in\left[h_{*}, h_{i, j}(t)[,\right. & t \in\left[t^{n}, t^{n+1}\right], \\
c_{\ell, i, j}\left(h_{i, j}(t), t\right)=c_{\ell, i, j}^{s, n+1}, & & \text { if } h_{i, j}^{n+1}>h_{i, j}^{n}, \\
c_{\ell, i, j}\left(\zeta, t^{n}\right) & =c_{\ell, i, j}^{n}(\zeta), & \zeta \in\left[t^{n}, t^{n+1}\right],
\end{array}
$$

where $h_{i, j}$ is a convex combination of the discrete sediment height between times $t^{n}$ and $t^{n+1}$ :

$h_{i, j}(t)=(1-\alpha(t)) h_{i, j}^{n}+\alpha(t) h_{i, j}^{n+1}, \quad \alpha(t)=\frac{t-t^{n}}{t^{n+1}-t^{n}}$

with $t \in\left[t^{n}, t^{n+1}\right]$. The solution of this system can be easily computed by distinguishing sedimentation from erosion. In the first case, the procedure consists in adding a block of thickness $h_{i, j}^{n+1}-h_{i, j}^{n}$ above the column at time $t^{n}$, in which the composition is given by the surface concentrations

$c_{\ell, i, j}^{n+1}(\zeta)=\left\{\begin{array}{ll}c_{\ell, i, j}^{n}(\zeta), & \zeta \in\left[h_{*}, h_{i, j}^{n}[,\right. \\ c_{\ell, i, j}^{s, n+1}, & \zeta \in\left[h_{i, j}^{n}, h_{i, j}^{n+1}\right],\end{array} \quad \ell=1, \ldots, L\right.$.

In the situation of erosion, one only has to truncate the column at time $t^{n}$ up to the sediment height $h_{i, j}^{n+1}$. Hence,

$c_{\ell, i, j}^{n+1}(\zeta)=c_{\ell, i, j}^{n}(\zeta), \quad \zeta \in\left[h_{*}, h_{i, j}^{n+1}[, \quad \ell=1, \ldots, L\right.$

This procedure is illustrated in Figure 9.

We are now ready to tackle the discretization of the conservation laws of the lithologies (58). The time derivative, written in the form (57), is approximated by

$$
\frac{\mathfrak{F}_{\ell, i, j}^{n+1}-\mathfrak{F}_{\ell, i, j}^{n}}{\Delta t}=\frac{1}{\Delta t}\left(\int_{h_{*}}^{h_{i, j}^{n+1}} c_{\ell, i, j}^{n+1}(\zeta) \mathrm{d} \zeta-\int_{h_{*}}^{h_{i, j}^{n}} c_{\ell, i, j}^{n}(\zeta) \mathrm{d} \zeta\right)
$$




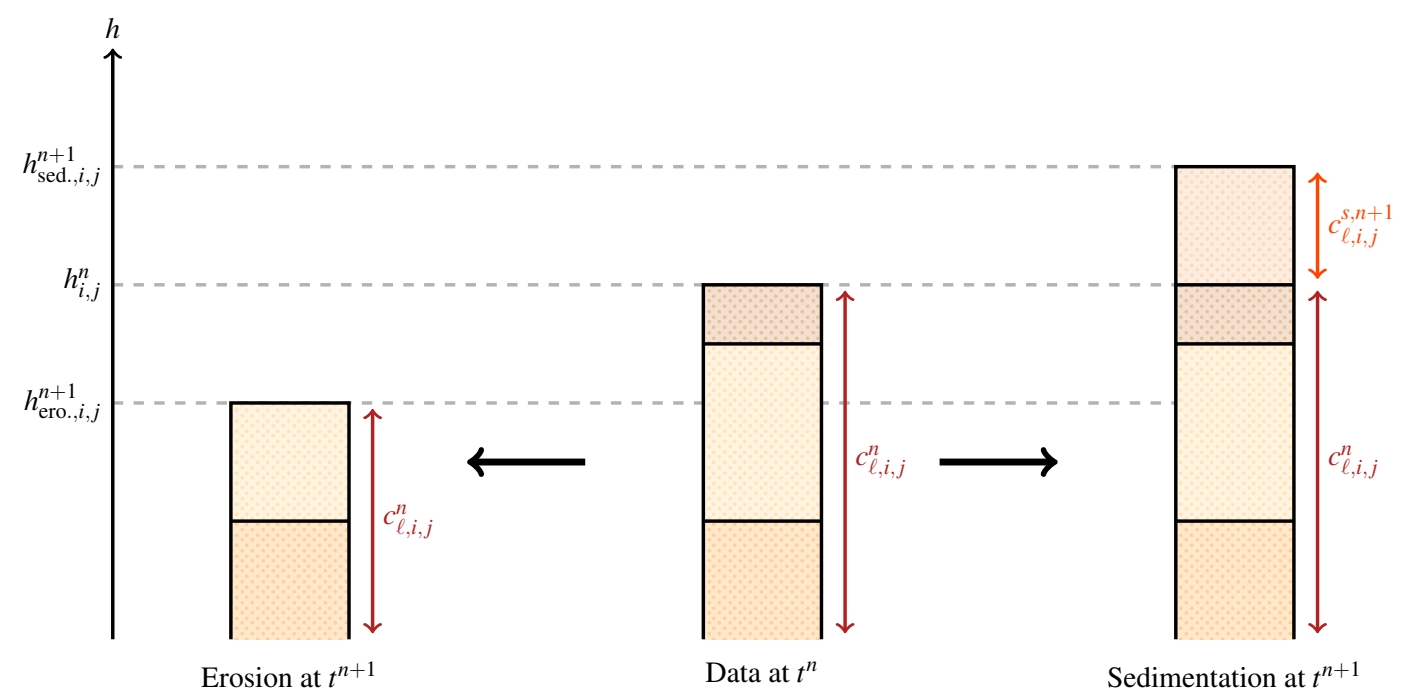

Fig. 9: Management of sediment columns.

Thanks to (62), this can be straightforwardly separated into two cases (sedimentation and erosion) as

$\frac{\mathfrak{F}_{\ell, i, j}^{n+1}-\mathfrak{F}_{\ell, i, j}^{n}}{\Delta t}= \begin{cases}c_{\ell, i, j}^{s, n+1} \frac{h_{i, j}^{n+1}-h_{i, j}^{n}}{\Delta t}, & \text { if } h_{i, j}^{n+1}>h_{i, j}^{n}, \\ \frac{1}{\Delta t} \int_{h_{i, j}^{n}}^{h_{i, j}^{n+1}} c_{\ell, i, j}^{n}(\zeta) \mathrm{d} \zeta, & \text { if } h_{i, j}^{n+1} \leqslant h_{i, j}^{n} .\end{cases}$

The lithology fluxes (59) can be written as $\mathbf{F}_{\ell}=c_{\ell}^{s} \vec{F}_{\ell}$, where $\overrightarrow{\mathscr{F}} \ell$ is the sediment flux (15b) used in the case of a single lithology. The only difference lies in the value of the diffusion coefficients, which may be different for each type of sediment. The associated approximation method consists in upwinding $\lambda c_{\ell}^{s}$ according to the sign of $\mathscr{F}=\overrightarrow{\mathscr{F}}_{\ell} \cdot \mathbf{n}$. It follows that the discretization of the full equation is given by

$$
\begin{aligned}
c_{\ell, i, j}^{s, n+1} \frac{\left(h_{i, j}^{n+1}-h_{i, j}^{n}\right)^{+}}{\Delta t} & +\frac{1}{\Delta t}\left(\int_{h_{i, j}^{n}}^{h_{i, j}^{n+1}} c_{\ell, i, j}^{n}(\zeta) \mathrm{d} \zeta\right)^{-} \\
+ & \frac{\left(\lambda F_{\ell}\right)_{i+1 / 2, j}^{n+1}-\left(\lambda F_{\ell}\right)_{i-1 / 2, j}^{n+1}}{\Delta x} \\
+ & \frac{\left(\lambda F_{\ell}\right)_{i, j+1 / 2}^{n+1}-\left(\lambda F_{\ell}\right)_{i, j-1 / 2}^{n+1}}{\Delta y}=0,
\end{aligned}
$$

where the discrete limited fluxes read

$$
\begin{aligned}
\left(\lambda F_{\ell}\right)_{i+1 / 2, j}^{n+1}= & \lambda_{i, j}^{n+1}\left(c_{\ell, i, j}^{s, n+1}\right)^{+}\left(\mathscr{F}_{\ell, i+1 / 2, j}^{n+1}\right)^{+} \\
& +\lambda_{i+1, j}^{n+1}\left(c_{\ell, i+1, j}^{s, n+1}\right)^{+}\left(\mathscr{F}_{\ell, i+1 / 2, j}^{n+1}\right)^{-}, \\
\left(\lambda F_{\ell}\right)_{i, j+1 / 2}^{n+1}= & \lambda_{i, j}^{n+1}\left(c_{\ell, i, j}^{s, n+1}\right)^{+}\left(\mathscr{F}_{\ell, i, j+1 / 2}^{n+1}\right)^{+} \\
& +\lambda_{i, j+1}^{n+1}\left(c_{\ell, i, j+1}^{s, n+1}\right)^{+}\left(\mathscr{F}_{\ell, i, j+1 / 2}^{n+1}\right)^{-} .
\end{aligned}
$$

The quantities $\mathscr{F}_{\ell, i+1 / 2, j}^{n+1}$ and $\mathscr{F}_{\ell, i, j+1 / 2}^{n+1}$ are then defined analogously to (34). In equations (64), we have deliberately taken the positive part of all surface concentrations. This enables us to show that these quantities remain nonnegative at each time-step.

The discretization of the complementarity equation (14c) follows exactly the same steps as for the case in a single lithology. It is not difficult to show that we still obtain

$\lambda_{i, j}^{n+1}=\min \left(1, \frac{\left.\Delta x \Delta y E_{i, j}+\right\rangle \lambda F\left\langle_{i, j}^{n+1}\right.}{\langle F\rangle_{i, j}^{n+1}}\right)$.

The difference is hidden in the definition of the outgoing and limited incoming fluxes, which now take the form

$$
\begin{array}{r}
\langle F\rangle_{i, j}^{n+1}=\sum_{\ell=1}^{L}\left(c_{\ell, i, j}^{s, n+1}\right)^{+}\left\{\Delta y\left[\left(\mathscr{F}_{\ell, i+1 / 2, j}^{n+1}\right)^{+}-\left(\mathscr{F}_{\ell, i-1 / 2, j}^{n+1}\right)^{-}\right]\right. \\
\left.+\Delta x\left[\left(\mathscr{F}_{\ell, i, j+1 / 2}^{n+1}\right)^{+}-\left(\mathscr{F}_{\ell, i, j-1 / 2}^{n+1}\right)^{-}\right]\right\}, \quad \text { (66) }
\end{array}
$$

and

$$
\begin{array}{r}
\rangle \lambda F\left\langle_{i, j}^{n+1}=\Delta y \sum_{\ell=1}^{L}\left\{\lambda_{i-1, j}^{n+1}\left(c_{\ell, i-1, j}^{s, n+1}\right)^{+}\left(\mathscr{F}_{\ell, i-1 / 2, j}^{n+1}\right)^{+}\right.\right. \\
\left.-\lambda_{i+1, j}^{n+1}\left(c_{\ell, i+1, j}^{s, n+1}\right)^{+}\left(\mathscr{F}_{\ell, i+1 / 2, j}^{n+1}\right)^{-}\right\} \\
+\Delta x \sum_{\ell=1}^{L}\left\{\lambda_{i, j-1}^{n+1}\left(c_{\ell, i, j-1}^{s, n+1}\right)^{+}\left(\mathscr{F}_{\ell, i, j-1 / 2}^{n+1}\right)^{+}\right. \\
\left.-\lambda_{i, j+1}^{n+1}\left(c_{\ell, i, j+1}^{s, n+1}\right)^{+}\left(\mathscr{F}_{\ell, i, j+1 / 2}^{n+1}\right)^{-}\right\} .
\end{array}
$$

In the case $\langle F\rangle_{i, j}^{n+1}=0$, we infer as previously that $\lambda_{i, j}^{n+1}=1$.

The closure equation (54) is approximated by the simple formula

$\sum_{\ell=1}^{L} c_{i, j}^{s, n+1}=1$ 
The last aspect to look at is the boundary conditions. Roughly speaking, we extend what has been done in the case of a single lithology. First, we consider the same equations (42) to take into account the water inflows. Instead of considering analogous ones for a global sediment inflow, we adapt one set of equations (43) for each lithology. To have a wellposed system, we also need to impose the discrete closure equation (68) on the boundary. Concerning the values in the four remaining ghost cells in the corners, we just have to consider the same equations as (44)-(45). Indeed, no concentrations nor columns are needed in these areas, as they only serve to compute local approximations of terms of the kind $|\nabla h|^{p-2}$.

\subsection{Numerical results}

We now illustrate the multilithological model with two test cases.

\subsubsection{Evolution of an estuary}

The first one stems from real-world data representing the evolution of an estuary in an Iranian region, at the Paleogene period. The domain of study measures $120 \mathrm{~km} \times 260 \mathrm{~km}$, and is divided into a marine and a continental part, the sea level being fixed at $H_{m}=0.6 \mathrm{~km}$. For this configuration, we assume sediments are made of a mixture of four lithologies, whose respective diffusion coefficients are detailed in Table 5 . The value of $\widetilde{K}$ is set to $10^{6} \mathrm{~km}^{2} / \mathrm{My}$. We also consider a constraint on the erosion process, which is characterized by a maximum erosion rate $E=0.008 \mathrm{~km} / \mathrm{My}$. Multiples sources of water and sediment inflows are prescribed, which is presented in Table 6 .

\begin{tabular}{ccccc}
\hline Lithology & $K_{m}$ & $K_{c}$ & $K_{g, m}$ & $K_{g, c}$ \\
\hline 1 & 4000 & 9000 & 25 & 1500 \\
2 & 11000 & 21000 & 60 & 1800 \\
3 & 13000 & 28000 & 75 & 2400 \\
4 & 24000 & 38000 & 200 & 3300 \\
\hline
\end{tabular}

Table 5: Diffusion coefficients value $\left(K_{m}\right.$ and $K_{c}$ in $\mathrm{km}^{1 / 2} / \mathrm{My}, K_{g, m}$ and $K_{g, c}$ in $\left.\mathrm{km}^{2} / \mathrm{My}\right)$.

The discretization of the domain consists of $48 \times 104$ cells, while the simulation time is set to $T=4 \mathrm{My}$. The exponent of the $p$-Laplacian in the lithology fluxes (59) is set to $p=2.5$. Concerning the sediment columns, we impose a maximum layers number of 100 , which already gives good approximations. We start the simulation with the initial time-step $\Delta t^{0}=10^{-10} \mathrm{My}$. This value may seem small, but is sometimes needed to start the simulation. Though if

\begin{tabular}{ccc}
\hline Boundary flux & $\Gamma_{b}$ & $\Gamma_{r}$ \\
\hline$\widetilde{\phi}$ & 360 & - \\
$\phi_{1}$ & 0.36 & 0.95 \\
$\phi_{2}$ & 0.18 & 0.095 \\
$\phi_{3}$ & 0.036 & 9.5 \\
$\phi_{4}$ & 0.018 & 5.0 \\
\hline
\end{tabular}

Table 6: Value of instantaneous boundary fluxes, integrated over $\partial \Omega\left(\right.$ in $\left.\mathrm{km}^{3} / \mathrm{My}\right)$.

such a case arises, these small values are no longer necessary for the rest of the simulation. The resolution of the linear systems arising at each Newton iteration is carried out using the BiCGStab [31] method from PETSc routines [3]. If the time iteration is accepted, we define the next time-step as $\Delta t^{n+1}=1.1 \Delta t^{n}$, up to the maximum value $5.10^{-3} \mathrm{My}$. On the contrary, if Newton's method fails to converge after 20 iterations, the time-step is rejected and is restarted with $\Delta t^{n}=0.5 \Delta t^{n}$.

The initial state of the system is displayed in Figure 10. One can see that the constraint is active on a large part of the domain, essentially in steep areas. The distribution of the different lithologies are also represented: for example lithology 4 is mainly located in the marine domain. The Figure 11 shows the state of the system after $4 \mathrm{My}$. We first observe that the diffusion has smoothed the topography. Moreover, the contrast within the diffusion coefficients leads to a break along the shoreline. Concentrations in lithologies have also undergone many changes. Looking again at lithology 4 , we notice that it seems concentrated in the pits in the center of the domain.

In order to have a more precise view of the internal structure of the sedimentary basin, we make a slice along the axe $x=84 \mathrm{~km}$. The result is shown in Figure 12. As lithology 4 is present from the beginning in this area and is also the one associated to the highest diffusion coefficients, it is not surprising to find it in the central pit. The remaining deposits are made of lithologies 1 and 2, as they are found in the surrounding areas. Also, on the left side we observe high concentrations in lithologies 3 and 4, which is due to a prescribed inflow of sediments nearby.

The Table 7 gathers numerical data obtained at the end of the simulation. In general, very few time-steps are refused for this test case. As for the configurations in the case of one lithology, a comparison between different preconditioners is made. We notice that the choice of the preconditionning method alters notably the computing time. In this case, ILU(2) seems to be the better option, whereas ILU(1) leads to higher refused time-steps and an important increase in the computing time. 


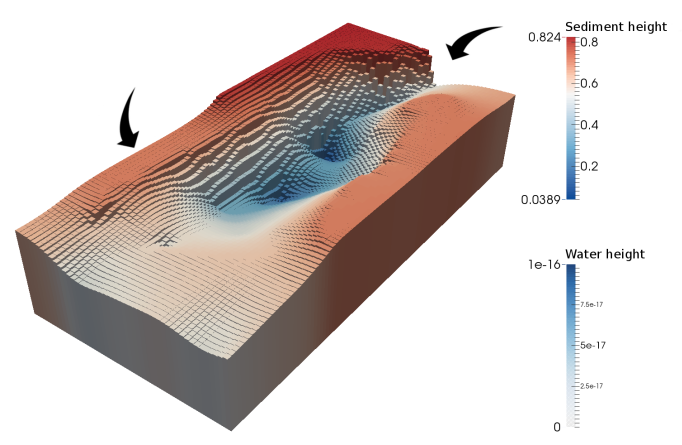

(a) Water and sediment height $(\mathrm{km})$.

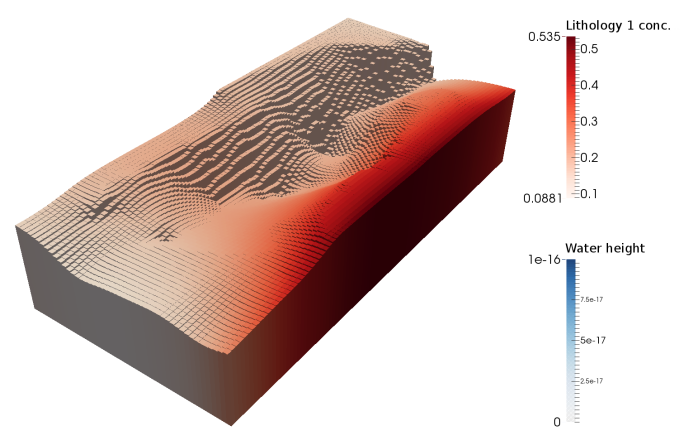

(c) Lithology 1 concentration.

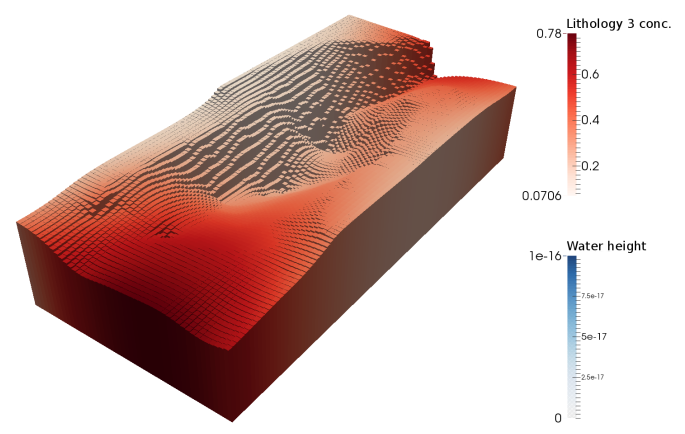

(e) Lithology 3 concentration.

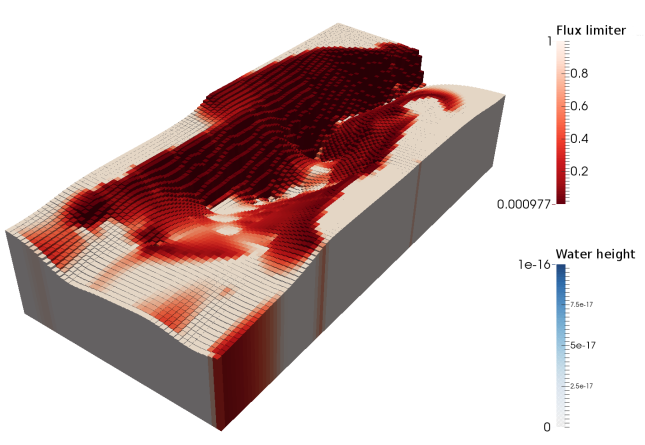

(b) Flux limiter.

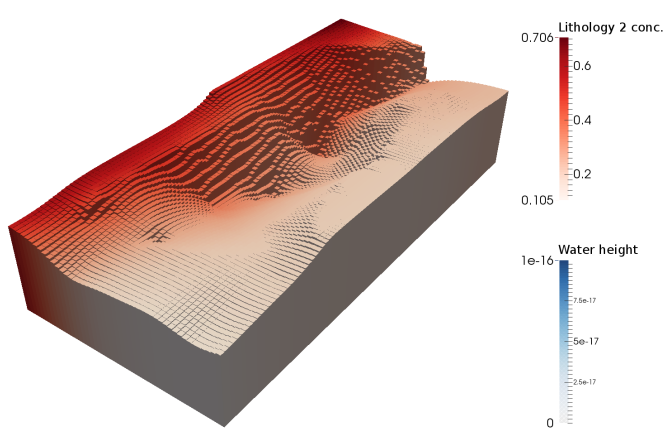

(d) Lithology 2 concentration.

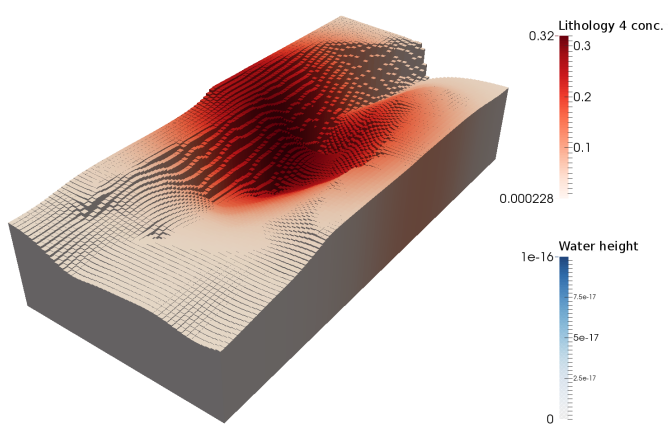

(f) Lithology 4 concentration.

Fig. 10: Initial data.

\subsubsection{Influence of bathymetry}

The second test case is aimed at capturing the influence of eustasy (variations of the sea level) on sedimentary deposit. We consider a domain of size $50 \mathrm{~km} \times 75 \mathrm{~km}$, discretized by a grid of $100 \times 150$ rectangular cells. The simulations runs over a duration of $T=320 \mathrm{My}$, using the exponent $p=2.5$ for the $p$-Laplacian. Water fluxes are neglected. The sediments are made up of three distinct lithologies, the gravity diffusion coefficients of which are given in Table 8.
The maximal erosion constraint is set to the value $E=$ $10^{-3} \mathrm{~km} / \mathrm{My}$. The sea level is imposed as a known function of time, represented in Figure 13. Its evolution can be divided into three cycles: (i) a long cycle from $30 \mathrm{My}$ to 130 My; (ii) a short cycle from 145 to $161 \mathrm{My}$; (iii) a "mixed" cycle from 203 My to 309 My.

The sediment columns are discretized by at most 100 layers. The stopping criterion for the Newton-min method is based on the $L^{\infty}$-residual of equations: $10^{-9} \mathrm{~km}$ for balance laws of lithologies and $10^{-5}$ for various complementarity 


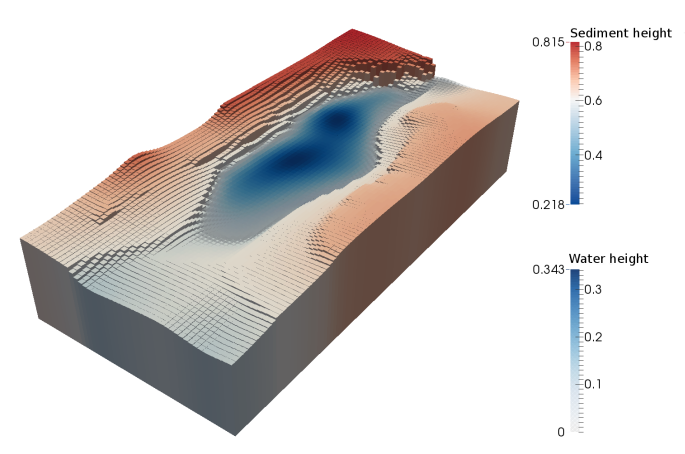

(a) Water and sediment heights $(\mathrm{km})$.

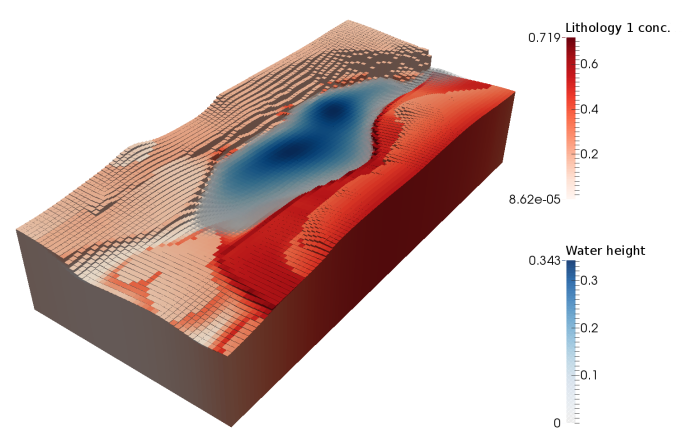

(c) Lithology 1 concentration.

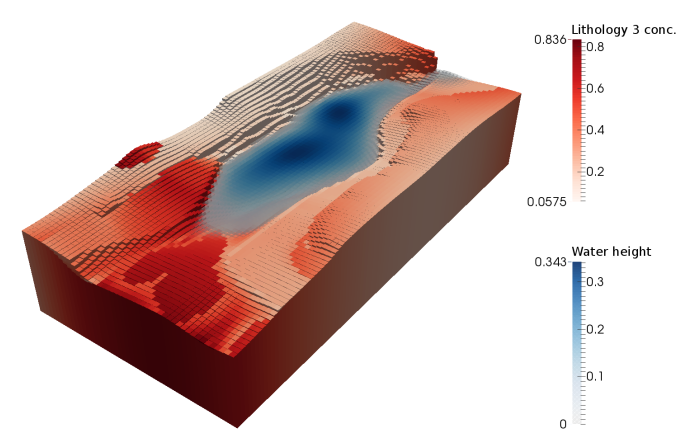

(e) Lithology 3 concentration.

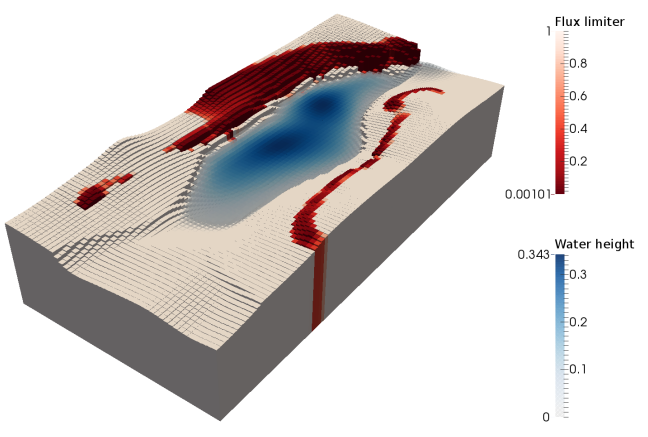

(b) Flux limiter.

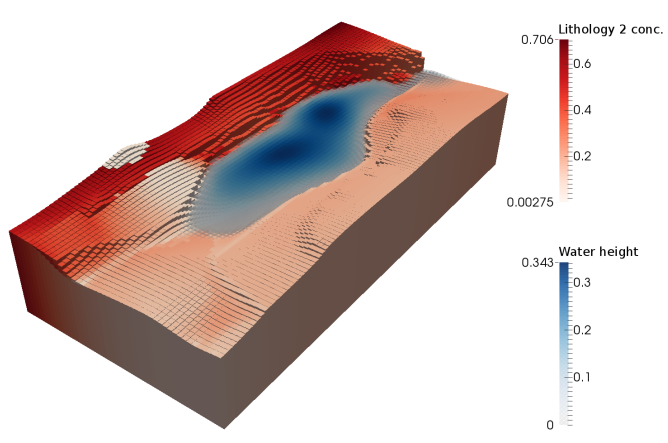

(d) Lithology 2 concentration.

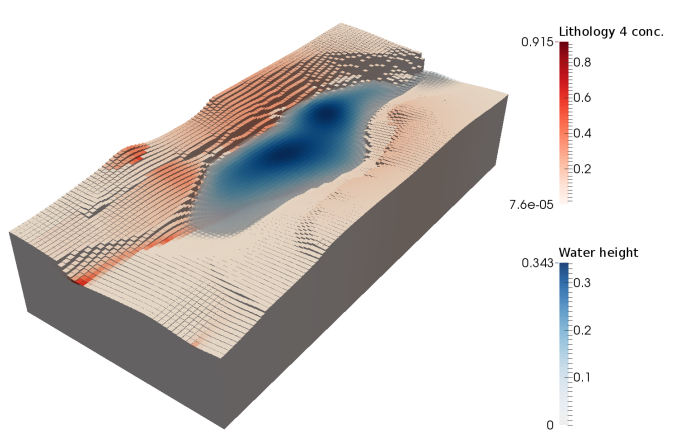

(f) Lithology 4 concentration.

Fig. 11: State at time $T=4 \mathrm{My}$.

\begin{tabular}{lcccc}
\hline \multirow{2}{*}{ Numerical data } & \multicolumn{4}{c}{ Preconditioner } \\
\cline { 2 - 5 } & $\mathrm{ILU}(1)$ & $\mathrm{ILU}(2)$ & $\mathrm{ILU}(3)$ & $\mathrm{ILU}(4)$ \\
\hline Accepted time-steps & 1216 & 1004 & 981 & 979 \\
Refused time-steps & 62 & 9 & 2 & 1 \\
Mean Newton iterations per time-step & 7.39 & 5.43 & 5.32 & 5.31 \\
Mean solver iterations per Newton iteration & 11.66 & 7.70 & 6.02 & 5.33 \\
CPU time (s) & 2171 & 1551 & 2063 & 2935 \\
\hline
\end{tabular}

Table 7: Numerical results. 


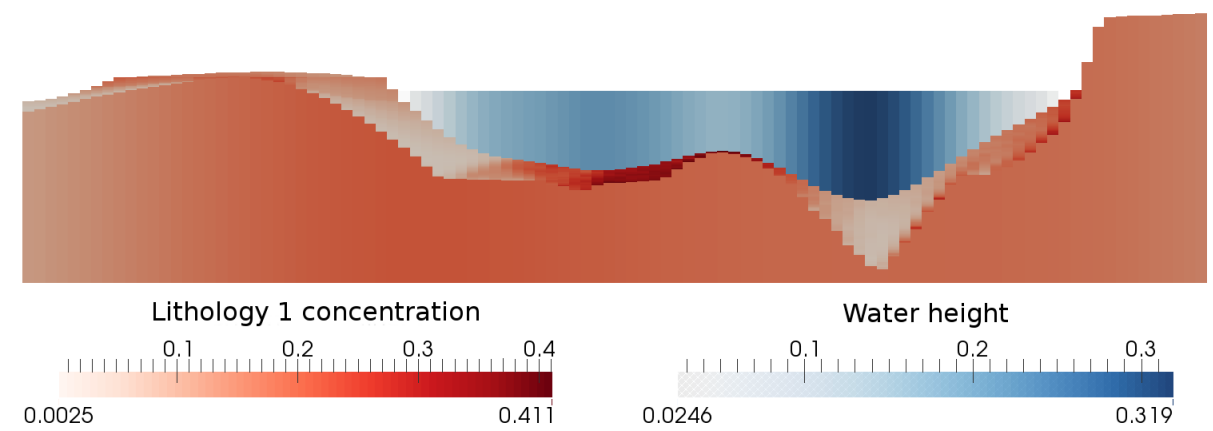

(a) Lithology 1 concentration.

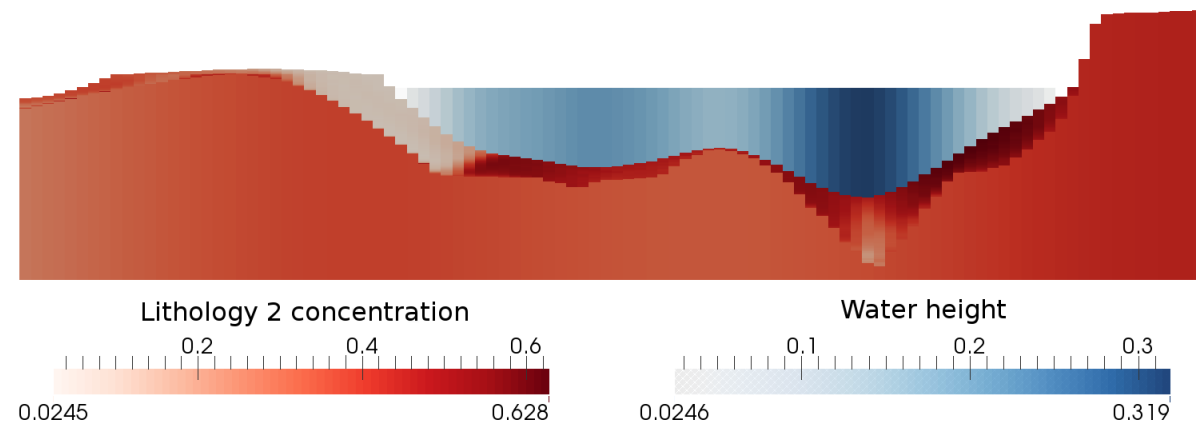

(b) Lithology 2 concentration.

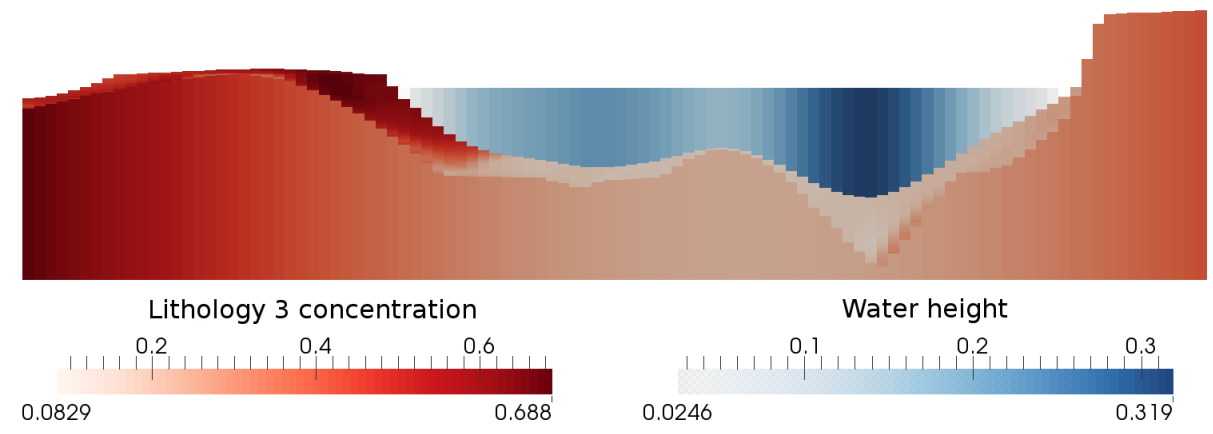

(c) Lithology 3 concentration.

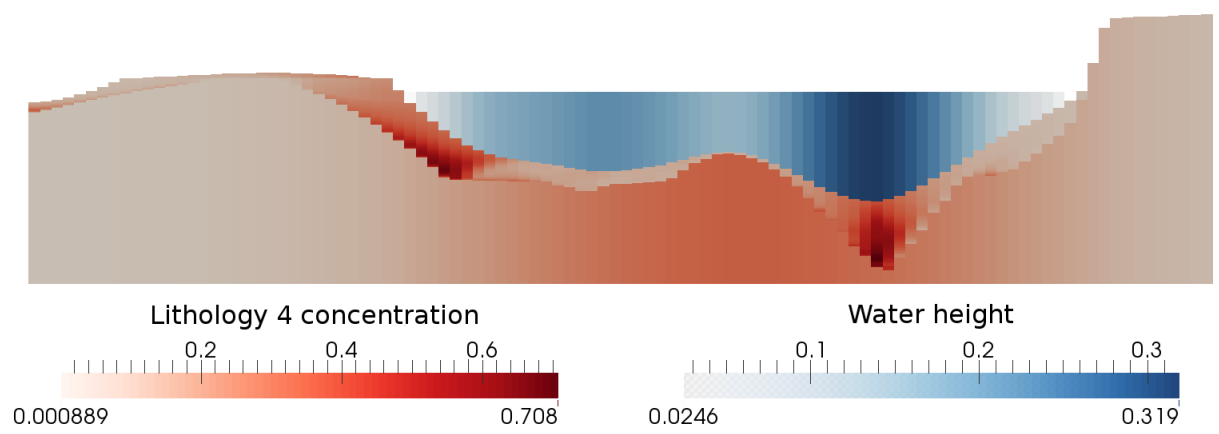

(d) Lithology 4 concentration.

Fig. 12: Slice at $x=84 \mathrm{~km}$ : lithology concentrations at time $T=4 \mathrm{My}$. 


\begin{tabular}{ccc}
\hline Lithologie & $K_{g, m}\left(\mathrm{~km}^{2} / \mathrm{Ma}\right)$ & $K_{g, c}\left(\mathrm{~km}^{2} / \mathrm{My}\right)$ \\
\hline 1 & 2 & 20 \\
2 & 4 & 40 \\
3 & 8 & 80 \\
\hline
\end{tabular}

Table 8: Gravity diffusion coefficients $K_{g, m}$ and $K_{g, c}$.

and closure equations. Linear systems are solved by means of the same algorithm and preconditioner as in $\$ 7.3 .1$. The management of time-steps $\Delta t^{n}$ is the same as in $\S 7.3 .1$.

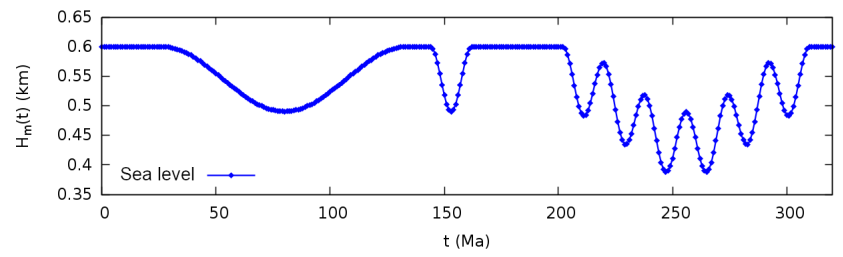

Fig. 13: Evolution of the sea level $H_{m}(t)$.

The inflows of sediments at the boundary are given by

$$
\begin{aligned}
& -\int_{0}^{T} \int_{0}^{L_{x}} \phi_{1}(x, 0, t) \mathrm{d} x \mathrm{~d} t=0.176 T \mathrm{~km}^{3} \\
& -\int_{0}^{T} \int_{0}^{L_{x}} \phi_{2}(x, 0, t) \mathrm{d} x \mathrm{~d} t=0.88 T \mathrm{~km}^{3} \\
& -\int_{0}^{T} \int_{0}^{L_{x}} \phi_{3}(x, 0, t) \mathrm{d} x \mathrm{~d} t=0.44 T \mathrm{~km}^{3} .
\end{aligned}
$$

The initial state of the system is displayed in Figure 14. We observe that the various strata are already there, with spacedependent concentrations in lithologies. At the end of the simulation, the state of the system is depicted in Figure 15. We observe that gravity diffusion has smoothed out the topography almost everywhere, except for the upper corners of the domain where the erosion constraint is still active. Besides, it is possible to notice a few geological structures emerging from the variations of different concentrations.

In order to better inspect those structures, a slice-cut is performed at $x=25 \mathrm{~km}$ and reported in Figures 16-17. At the beginning, lithology 3 whose diffusion coefficients are the strongest is found mainly at the bottom. The other two lithologies, which move at a lower speed, share the remaining space. After a while, the deposition structures appearing on the lithology concentrations can be related to sediments deposited during the different eustasy cycles, as indicated in Figure 17. We can then distinguish the marks left in the short cycle and the long cycle in Figure 16. The mixed cycle is more difficult to single out.

Finally, some numerical data in relation with the simulation are gathered in Table 9. It can be seen that a few time-steps have been refused and had to be restarted. This non-convergence is due to a cyclic behavior, which occurs when $\Delta t$ is too large.

\begin{tabular}{lr}
\hline Numerical data & Value \\
\hline Accepted time-steps & 16644 \\
Refused time-steps & 250 \\
Mean Newton iterations per time-step & 4.74 \\
Mean solver iterations per Newton iteration & 5.28 \\
CPU time (h) & 9.72 \\
\hline
\end{tabular}

Table 9: Numerical results.

\section{Conclusion}

The present contribution is a logical sequel to [13, 14, 2022]. We have successfully embedded two additional physical processes to the model of DionisosFlow, while maintaining the foundational aspects of the previous work. The two novelties under consideration - nonlinear gravity diffusion flux for sediment transport and coupling between sediments and water effects - have required tremendous changes in the numerical scheme. The new scheme, built upon the old one and the ideas of Andreianov et al. [1] for the $p$-Laplacian, allows us to preserve the physical bounds of various unknowns and also guarantees the existence of a discrete solution. The simulation results it provides on realistic test cases have been deemed very satisfactory by geologists and demonstrate its overall efficiency.

There are many improvements and extensions that could possibly be envisaged. On the one hand, more optimized numerical treatments could be tried in an attempt to consolidate the code. More specifically, we believe that block preconditioning (to speed up the resolution of linear systems) and step-control in Newton's method (to avoid cyclic behavior and other failures in the resolution of nonlinear systems) would significantly enhance the performance of the scheme. We have unfortunately not had time to deepen those issues. On the other hand, more physical processes could be included into the model. In the short term, adding source terms in the balance laws would enable us to model rain, evaporation and production of carbonates. In the middle term, it would be reasonable to incorporate subsidence and compaction. In the long run, it might be challenging to work out a model for marine hyperpycnal/hypopycnal flows in the zones mixing river water and standing ocean water.

\section{References}

1. Andreianov, B., Boyer, F., Hubert, F.: Finite volume schemes for the $p$-Laplacian on Cartesian meshes. M2AN 38(6), 931-959 (2004). DOI 10.1051/m2an:2004045 


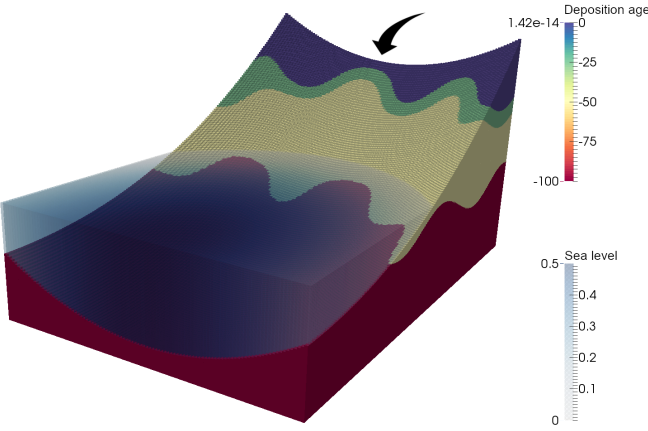

(a) Deposition age.

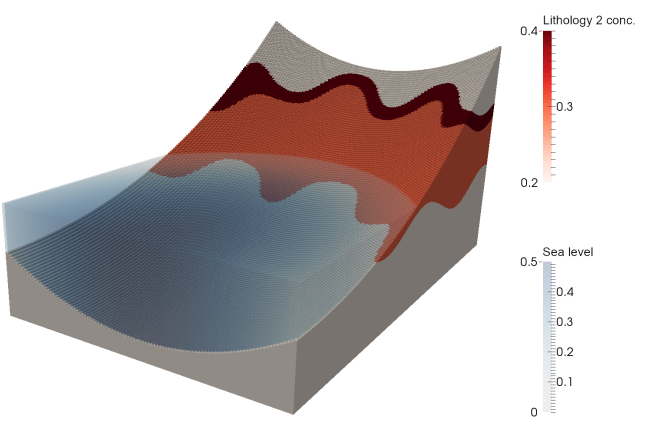

(c) Concentration of lithology 2 .

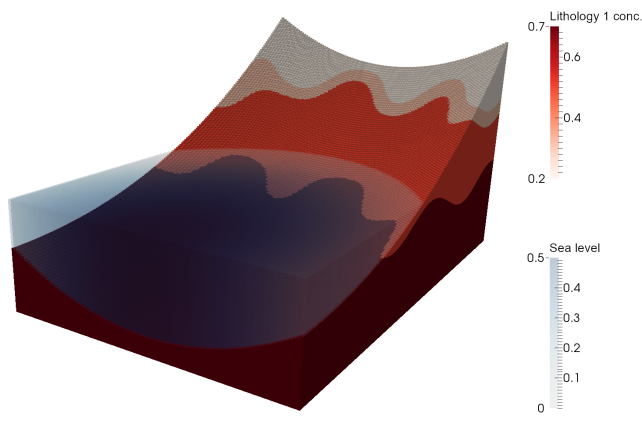

(b) Concentration of lithology 1 .

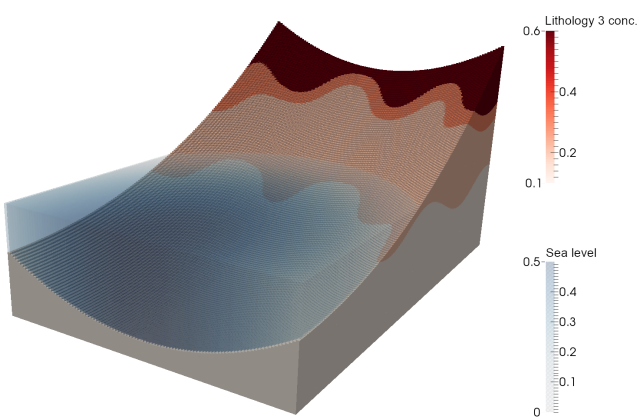

(d) Concentration of lithology 3 .

Fig. 14: Deposition age and lithology concentrations at time $T=0 \mathrm{My}$.

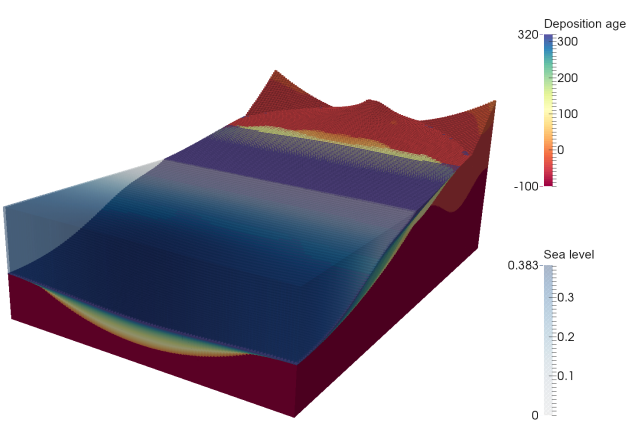

(a) Deposition age.

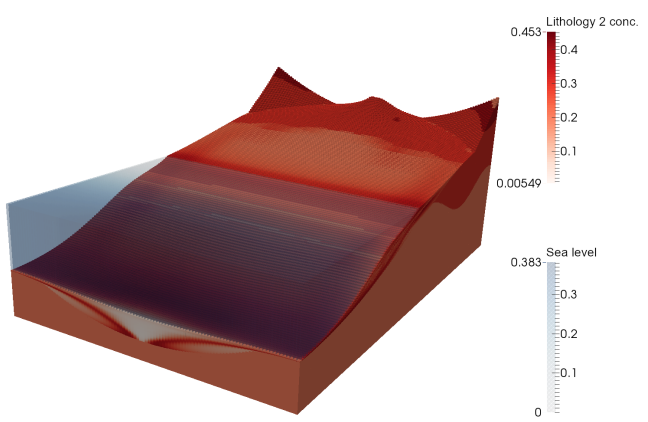

(c) Concentration of lithology 2 .

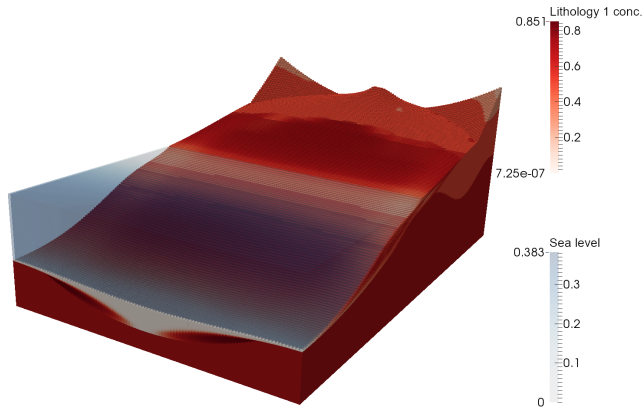

(b) Concentration of lithology 1 .

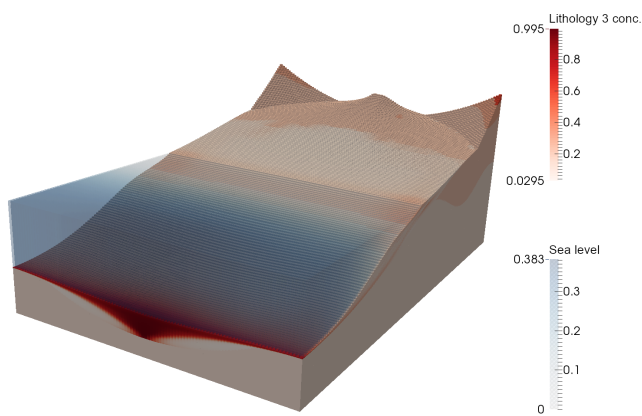

(d) Concentration of lithology 3 .

Fig. 15: Deposition age and lithology concentrations at time $T=320 \mathrm{My}$. 


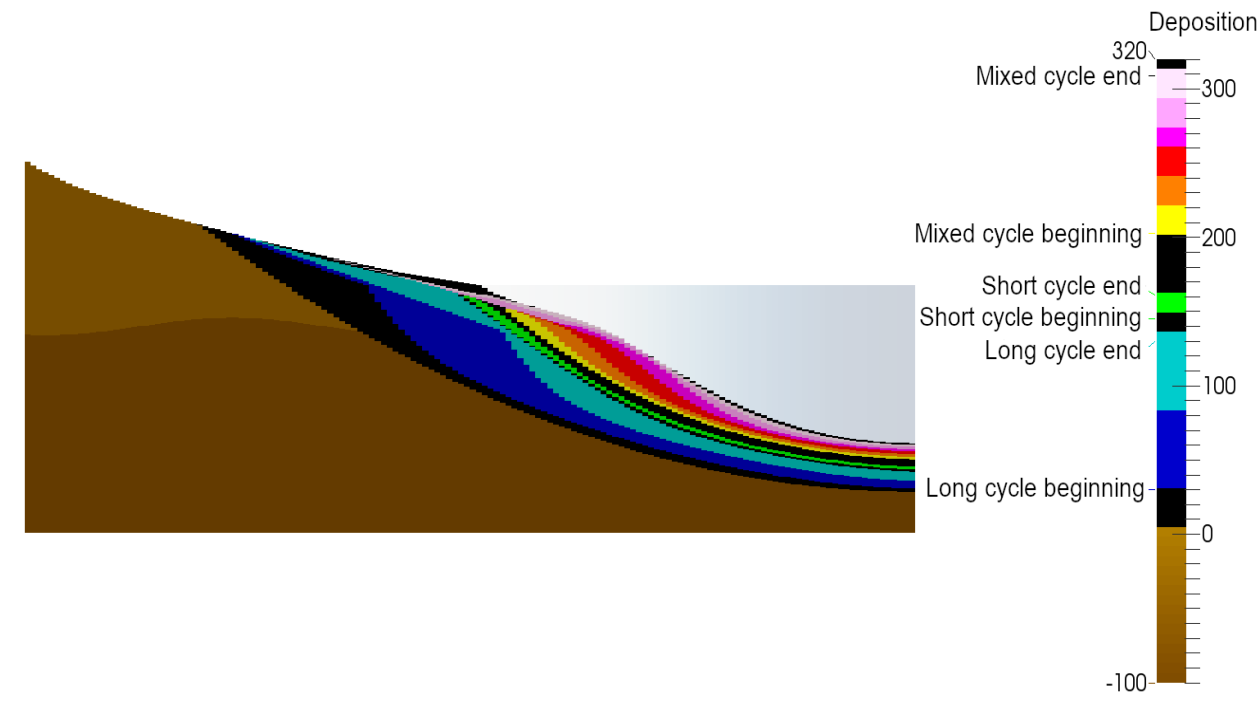

Fig. 16: Influence of eustasy on sedimentary deposit.

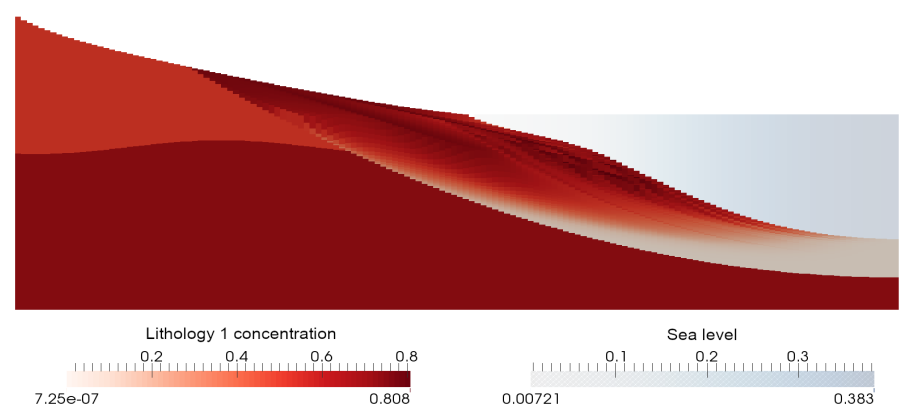

(a) Concentration of lithology 1 .

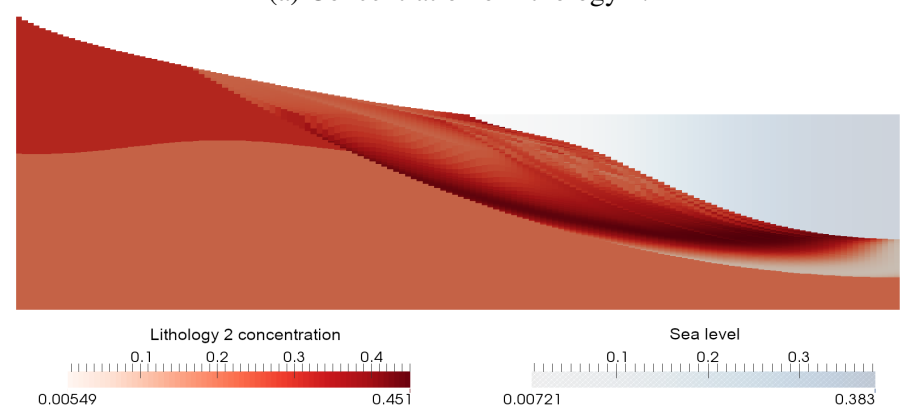

(b) Concentration of lithology 2 .

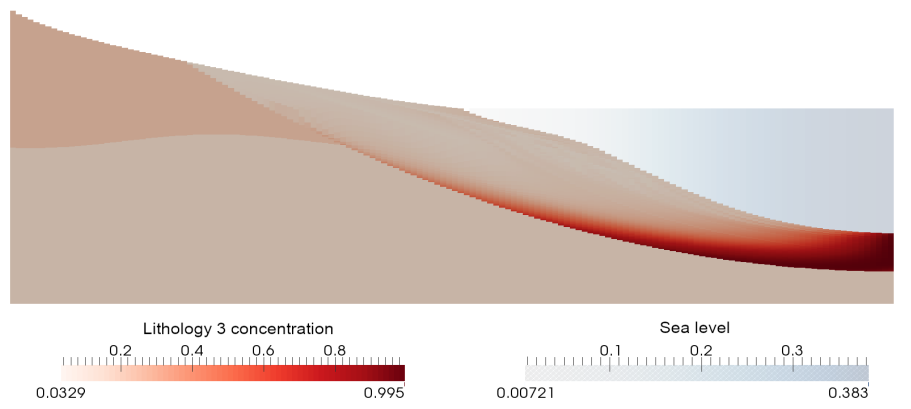

(c) Concentration of lithology 3 .

Fig. 17: Slice at $x=25 \mathrm{~km}$ : Lithology concentrations at time $T=320 \mathrm{My}$. 
2. Audusse, E., Boyaval, S., Goutal, N., Jodeau, M., Ung, P.: Numerical simulation of the dynamics of sedimentary river beds with a stochastic Exner equation. ESAIM: Proc. 48, 321-340 (2015). DOI 10.1051/proc/201448015

3. Balay, S., Abhyankar, S., Adams, M.F., Brown, J., Brune, P., Buschelman, K., Dalcin, L., Eijkhout, V., Gropp, W.D., Kaushik, D., Knepley, M.G., McInnes, L.C., Rupp, K., Smith, B.F., Zampini, S., Zhang, H., Zhang, H.: PETSc users manual. Tech. Rep. ANL-95/11 - Revision 3.7, Argonne National Laboratory (2016). URL http://www.mcs.anl.gov/petsc

4. Barenblatt, G.I.: On self-similar motions of a compressible fluid in a porous medium. Prikl. Mat. Mech. 16(6), 679-698 (1952)

5. Ben Gharbia, I.: Résolution de problèmes de complémentarité. : Application à un écoulement diphasique dans un milieu poreux. Ph.D. thesis, Université Paris Dauphine (Paris IX) (2012). URL http://tel.archives-ouvertes.fr/tel-00776617

6. Birnir, B., Smith, T.R., Merchant, G.E.: The scaling of fluvial landscapes. Computers \& Geosciences 27(10), 1189-1216 (2001). DOI 10.1016/S0098-3004(01)00022-X

7. Cancès, C., Granjeon, D., Peton, N., Tran, Q.H., Wolf, S.: Numerical scheme for a stratigraphic model with erosion constraint and nonlinear gravity flux. In: FVCA 8 - 2017 - International Conference on Finite Volumes for Complex Applications VIII, Proceedings in Mathematics \& Statistics, vol. 200, pp. 327-335. Springer (2017). DOI 10.1007/978-3-319-57394-6_35

8. Cordier, S., Lê, M.H., Morales de Luna, T.: Bedload transport in shallow water models: Why splitting (may) fail, how hyperbolicity (can) help. Adv. Water Res. 34(8), 980-989 (2011). DOI 10.1016/j.advwatres.2011.05.002

9. Delestre, O., Cordier, S., James, F., Darboux, F.: Simulation of rain-water overland-flow. In: J.G.L. E. Tadmor, A. Tzavaras (eds.) 12th International Conference on Hyperbolic Problems, Proceedings of Symposia in Applied Mathematics, vol. 67, pp. 537546. American Mathematical Society, College Park, United States (2008). URL https://hal.archives-ouvertes.fr/hal-00343721

10. Di Benedetto, E., Herrero, M.A.: Non-negative solutions of the evolution $p$-Laplacian equation. initial traces and Cauchy problem when $1<p<2$. Trans. Amer. Math. Soc. 314(1), 187-224 (1989). DOI 10.1090/S0002-9947-1989-0962278-5

11. Droniou, J.: Degrés topologiques et applications (2006). URL http://users.monash.edu.au/ jdroniou/docs/degre.pdf

12. Droniou, J.: Finite volume schemes for fully non-linear elliptic equations in divergence form. ESAIM: Math. Model. Numer. Anal. 40(6), 1069-1100 (2006). DOI 10.1051/m2an:2007001

13. Eymard, R., Gallouët, T., Gervais, V., Masson, R.: Existence and uniqueness of a weak solution to a stratigraphic model. In: M. Feistauer, V. Dolejší, P. Knobloch, K. Najzar (eds.) Numerical Mathematics and Advanced Applications, pp. 278-287. Springer, Berlin (2004). DOI 10.1007/978-3-642-18775-9-25

14. Eymard, R., Gallouët, T., Gervais, V., Masson, R.: Convergence of a numerical scheme for stratigraphic modeling. SIAM J. Numer. Anal. 43(2), 474-501 (2005). DOI 10.1137/S0036142903426208

15. Eymard, R., Gallouët, T., Ghilani, M., Herbin, R.: Error estimates for the approximate solutions of a nonlinear hyperbolic equation given by finite volume schemes. IMA J. Numer. Anal. 18(4), 563594 (1998). DOI 10.1093/imanum/18.4.563

16. Eymard, R., Gallouët, T., Granjeon, D., Masson, R., Tran, Q.H.: Multi-lithology stratigraphic model under maximum erosion rate constraint. Int. J. Numer. Meth. Eng. 60(2), 527-548 (2004). DOI 10.1002/nme.974

17. Eymard, R., Gallouët, T., Herbin, R.: Finite volume methods. In: P.G. Ciarlet, J.L. Lions (eds.) Techniques of Scientific Computing (Part 3), Handbook of Numerical Analysis, vol. VII, pp. 713-1018. North-Holland, Elsevier, Amsterdam (2000). DOI $10.1016 / \mathrm{S} 1570-8659(00) 07005-8$
18. Eymard, R., Gallouët, T., Herbin, R.: Cell centred discretisation of non linear elliptic problems on general multidimensional polyhedral grids. J. Numer. Math. 17(3), 173-193 (2009). DOI 10.1515/JNUM.2009.010

19. Freeman, T.G.: Calculating catchment area with divergent flow based on a regular grid. Computers \& Geosciences 17(3), 413422 (1991). DOI 10.1016/0098-3004(91)90048-I

20. Gervais, V.: Étude et simulation d'un modèle stratigraphique multi-lithologique sous contrainte de taux d'érosion maximal. Ph.D. thesis, Université de Provence, Aix-Marseille I (2004). URL http://tel.archives-ouvertes.fr/tel-01445562

21. Gervais, V., Masson, R.: Mathematical and numerical analysis of a stratigraphic model. ESAIM: Math. Model. Numer. Math. 38(4), 585-611 (2004). DOI 10.1051/m2an:2004035

22. Gervais, V., Masson, R.: Numerical simulation of a stratigraphic model. Comput. Geosci. 12(2), 163-179 (2008). DOI 10.1007/s10596-007-9076-4

23. Graf, W.H., Altinakar, M.S.: Hydraulique fluviale: Écoulement et phénomènes de transport dans les canaux à géométrie simple, Traité de Génie Civil, vol. 16. Presses polytechniques et universitaires romandes (2000)

24. Granjeon, D.: Modélisation stratigraphique déterministe: Conception et applications d'un modèle diffusif 3-d multilithologique. Ph.D. thesis, Université de Rennes I (1996). URL https://geosciences.univ-rennes1.fr/IMG/pdf/Granjeon.pdf

25. Holmgren, P.: Multiple flow direction algorithms for runoff modelling in grid based elevation models: An empirical evaluation. Hydrological Processes 8(4), 327-334 (1994). DOI 10.1002/hyp.3360080405

26. Kamin, S., Vázquez, J.L.: Fundamental solutions and asymptotic behaviour for the $p$-Laplacian equation. Rev. Mat. Iberoamericana 4(2), 339-354 (1988)

27. Kavian, O.: Introduction à la théorie des points critiques et applications aux problèmes elliptiques, Mathématiques \& Applications, vol. 13. Springer-Verlag (1993)

28. Peton, N.: Étude et simulation d'un modèle stratigraphique advecto-diffusif non-linéaire avec frontières mobiles. Ph.D. thesis, Université Paris-Saclay (2018)

29. Quinn, P., Beven, K., Chevallier, P., Planchon, O.: The prediction of hillslope flow paths for distributed hydrological modelling using digital terrain models. Hydrological Processes 5(1), 59-79 (1991). DOI 10.1002/hyp.3360050106

30. Rivenæs, J.C.: Application of a dual-lithology, depth-dependent diffusion equation in stratigraphic simulation. Basin Research 4(2), 133-146 (1992). DOI 10.1111/j.1365-2117.1992.tb00136.x

31. Saad, Y.: Iterative Methods for Sparse Linear Systems, 2nd edn. Society for Industrial and Applied Mathematics (2003). DOI $10.1137 / 1.9780898718003$

32. Tarboton, D.G.: A new method for the determination of flow directions and upslope areas in grid digital elevation models. Water Resources Research 33(2), 309-319 (1997). DOI 10.1029/96WR03137 